ANL - PHY $--87-2$

DE87 011651

Physics Division

Argonne National Laboratory

9700 South Cass Avenue

Argonne, Illinois 60439-4843

\title{
QUARKS, QCD AND THE REAL WORLD OF EXPERIMENTAL DATA
}

Lectures Given at Argonne National Laboratory

\author{
by \\ Harry J. Lipkin ${ }^{*}$
}

July 1987

\section{MASTER}

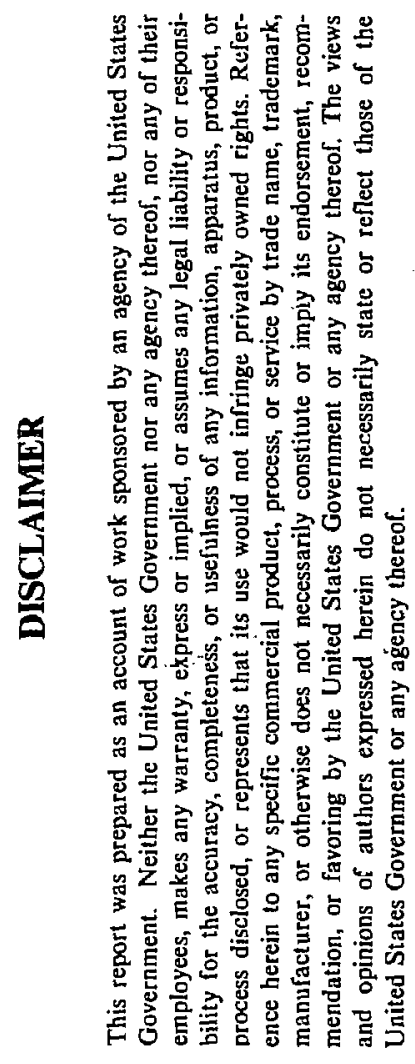

*1985-86 Argonne Fellow. Permanent address: Department of Physics, Weizmann Institute of Science, 76100 Rehovot, Israel 


\section{EDITOR'S PREFACE}

is a graduate student just entering the research community in theoretical nuclear physics, I was fortunate enough to be able to attend the lecture series entitled "Quarks, QCD and the Real World of Experimental Data", by Dr. Harry Lipkin. This publication is the result of notes that I took during that series. Dr. Lipkin's lectures were clear discussions of problems of current interest to both nuclear and particle physicists alike. I hope that my notes do justice to the lectures that Dr. Lipkin gave. My desire is that this document serve as a clear introduction for those new to the topics covered, and as a nice discussion of current interest for those more familiar with the subject. Accordingly, as ediror, I have inserted some helpful references in the text.

I would like to thank Dr. Lipkin for his help in preparing this document. I would also like to thank Ms. Donna Nelson for her help in typing the manuscript from my longhand notes, and Mr. Dave Kurth for the production of the figures for the publication.

C. G. Fasano

July 1987 


\section{TABLE OF CONTENTS}

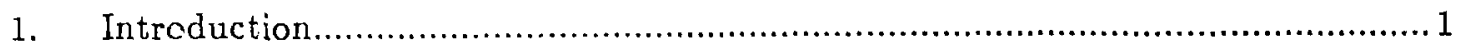

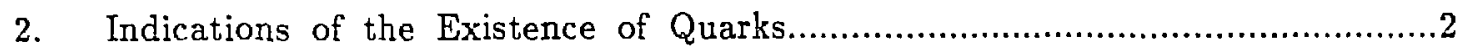

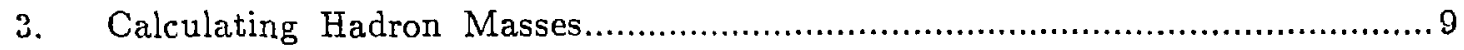

3.1 Flavor dependence................................................................. 10

3.2 Spin dependence.................................................................. 11

4. Additional Evidence of Substructure ... the Antiproton.................................12

4.1 Quark rearrangement............................................................13

4.2 The Fermi-Yang-Sakata model.............................................. 15

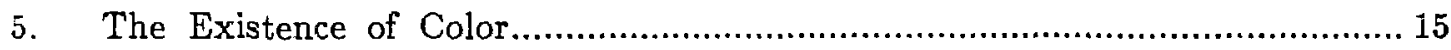

5.1 The saturation problem........................................................... 15

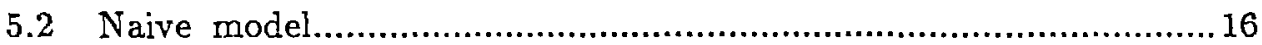

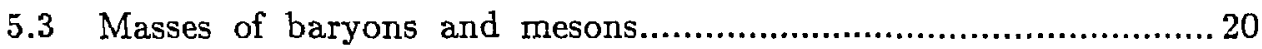

5.4 Another look at color........................................................... 23

6. Hadron Masses and the Hyperfine Interaction...........................................30

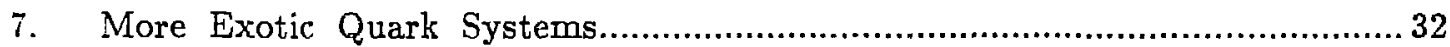

7.1 New physics in multiquark systems..........................................37

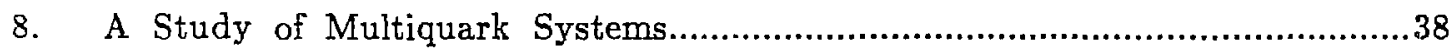

8.1 Multiquark systems from hadrons...............................................42

8.2 Weakly bound two meson systems...........................................50

9. Weak Interactions in the Quark Model Calculating $g_{\mathrm{A}} / \mathrm{g}_{\mathrm{V}} \ldots \ldots \ldots \ldots \ldots \ldots \ldots \ldots \ldots$

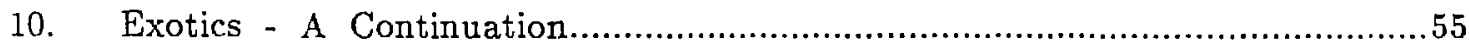

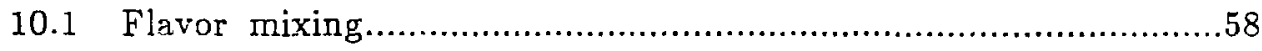




\section{Introduction}

At this time, we belleve that hadrons are composed of constituent particles called quarks, in a color fleld. Color is the "charge" that the quarks carry, and QCD (quantum chromodynamics) is the theory that describes how the quarks interact. QCD provides a description of the strong interaction. We begin by discussing how the experimental evidence forced QCD upon us.

It is interesting to begin by contrasting the development of theorles of the strong interaction with the development of theories of the electroweak interaction. Electroweak theory has developed with a "standard mode1" always in mind, (from Fermi's beta decay through QED). ExperimentalIsts have looked to verify the curient "standard model". As more data became avallable, the standard model ras simply modifled and extended, 1.e., Ferml's theory was extended to include Gamow-Teller transitions etc.

In the case of strong interaction theory, in contrast, there has never been a "standaid model". Inftially, protons and neutrons were the elementary constituents. Later there were meson exchanges and pion clouds. St111 later, quarks and color flelds were proposed: Rather than extending old models of strong Interactions, completely new 1deas supplanted the old. Theorists have been dragged klcking and screaming into the real world. Hadron physics today is much like superconductivity was in the 1950's. In the 50's, everyone belleved that the fundamental description of superconductivity was contalned in QED. Unfortunately though, the QED description of condensed natter was so complex that you could not actually calculate anything from first principles. BCS theory finally came along to tell us how to calculate, but still not from flrst princlples and the QED Lagrang lan . 
In the case of hadron physics, we have QCD as a description of the fundamental strong interaction. But like condensed matter physics with QED in the $1950^{\circ} \mathrm{s}$, it is too complicated to allow us to calculate anything very well. We need some type of model that approximates QCD to get properties of the theory out.

\section{Indications of the Existence of Quarks.}

The ratios of the nuclear magnetic moments of the proton and neutron gave an early indication that the proton and neutron were not elementary particles, but were actually composite.

Let us assume that the proton and neutron are composite particles, composed of constituents called quarks. Assume that $p$ and $n$ are composites of 3 quarks each in the following way:

$$
\begin{aligned}
& p=\text { uud } \\
& n=d d u
\end{aligned}
$$

where $u$ and $\dot{d}$ are quarks with the following properties

$$
\begin{array}{lll}
\text { u "up" } & \mathrm{q}_{\mathrm{u}}=+\frac{2}{3} \mathrm{e} & \mathrm{I}=1 / 2 \\
\mathrm{~d} \text { "down" } & \mathrm{q}_{\mathrm{d}}=-\frac{1}{3} \mathrm{e} & \mathrm{I}=1 / 2
\end{array}
$$

The $u$ and $d$ quarks form an isospin doublet. Let us calculate the ratio of the magnetic moments of the proton and neutron. Under the assumption that the proton and neutron are composites in the way described, the magnetic moment may be defined for the proton or neutron as 


$$
\stackrel{+}{\mu}=\sum_{1=1} \mu_{1} \stackrel{+}{S}_{1} \quad 1 \text { is sum over quarks }
$$

where $\mu_{1}$ is the magnetic moment of the $1^{\text {th }}$ quark and $J_{1}$ is the spin of the $1^{\text {th }}$ quark. We have assumed that there is no contribution due to the orbital motion of the quarks ( $1 . e$. we have essentlally put them in the $\ell=0$ s-state). In the case of the proton and neutron, there are only two types of quãiks, so

$$
\vec{\mu}=\mu_{u} \vec{S}_{u}+\mu_{d} \vec{S}_{d}
$$

where

$\mu_{u} \quad$ is the magnetic moment of the u quark
$\mu_{d} \quad$ is the magnetic moment of the d quark
$\vec{S}_{u}$
$\vec{S}_{d}$

The ratio of the proton magnetic moment to the neutron magnetic moment is given by

where

$$
\frac{\mu_{p}}{\mu_{n}}=\frac{\left\langle p+\left|\mu_{u} s_{z u}+\mu_{d} S_{z d}\right| p \uparrow\right\rangle}{\left\langle n \uparrow\left|\mu_{u} S_{z u}+\mu_{d} S_{z d}\right| n \uparrow\right\rangle}
$$

$$
\begin{aligned}
& S_{z u} \quad \text { is the } z \text { component of the u quark spin } \\
& S_{z d} \quad \text { is the } z \text { component of the d quark spin }
\end{aligned}
$$

The 1sospin properties of $p$ and $n$, and $u$ and $d$, can be used to rewrite the ratio as 
4

$$
\frac{\mu_{p}}{\mu_{n}}=\frac{\left\langle p+\left|\mu_{u} S_{z u}+\mu_{d} S_{z d}\right| p \uparrow\right\rangle}{\left\langle p+\left|\mu_{d} S_{z u}+\mu_{u} S_{z d}\right| p \uparrow\right\rangle}
$$

If we make the additional assumption that the magnetic moment of each quark is simply given by the charge of the quark times some fundamental moment,

$$
\begin{aligned}
& \mu_{u}=+\frac{2}{3} \mu \\
& \mu_{d}=-\frac{1}{3} \mu
\end{aligned}
$$

with $\mu$ as the fundamental moment, we can rewrite the ratio again is

$$
\frac{\mu_{p}}{\mu_{n}}=\frac{\left\langle p \uparrow\left|2 s_{z u}-s_{z d}\right| p \uparrow\right\rangle}{\left\langle p+\left|2 s_{z d}-s_{z u}\right| p \uparrow\right\rangle}
$$

It is convenient for our purposes to rearrange this expression as

$$
\frac{\mu_{p}}{\mu_{n}}=\frac{\left\langle p+\left|\frac{1}{2}\left(s_{z u}+s_{z d}\right)+\frac{3}{2}\left(s_{z u}-s_{z d}\right)\right| p \uparrow\right\rangle}{\left\langle p+\left|\frac{1}{2}\left(s_{z u}+s_{z d}\right)-\frac{3}{2}\left(s_{z u}-s_{z d}\right)\right| p \uparrow\right\rangle}
$$

with

$$
\begin{aligned}
& \left\langle p+\left|\frac{1}{2}\left(S_{z u}+s_{z d}\right)\right| p \uparrow\right\rangle \\
& =\frac{1}{2}\left\langle p+\left|s_{z}\right| p \uparrow\right\rangle \\
& =\frac{1}{4}
\end{aligned}
$$

we get

$$
\frac{\stackrel{u}{p}_{p}}{\vec{u}_{n}}=\frac{\frac{1}{4}+\frac{3}{2}\left\langle p+\left|\left(s_{z u}-s_{z d}\right)\right| p \uparrow\right\rangle}{\frac{1}{4}-\frac{3}{2}\left\langle p+\left|\left(s_{z u}-s_{z d}\right)\right| p \uparrow\right\rangle}
$$


and define

$$
\left\langle p \uparrow\left|\left(s_{z u}-s_{z d}\right)\right| p \uparrow\right\rangle
$$

as

$$
\left\langle\left(s_{z u}-s_{z d}\right)\right\rangle
$$

to get a simpler expression

$$
\frac{u_{p}}{u_{n}}=\frac{\frac{1}{4}+\frac{3}{2}\left\langle\left(s_{z u}-s_{z d}\right)\right\rangle}{\frac{1}{4}-\frac{3}{2}\left\langle\left(s_{z u}-s_{z d}\right)\right\rangle}
$$

Using the Wigner-Eckart Theorem (see Quantum Mechanics, Merzbacher, p. 403)

$$
\left\langle\mathrm{V}_{\mathrm{z}}\right\rangle=\frac{(\mathrm{V} \cdot \mathrm{J}) \mathrm{J}_{\mathrm{z}}}{\mathrm{J}(\mathrm{J}+1)}
$$

this allows us to write

$$
\begin{aligned}
& \left\langle\left(s_{u}-s_{d}\right)_{z}\right\rangle=\frac{\left\langle\left(\vec{S}_{u}-\vec{S}_{d}\right) \cdot \vec{J}\right\rangle J_{z}}{j(j+1)} \\
& =\frac{\left\langle\left(\vec{S}_{u}-s_{d}\right) \cdot\left(\vec{s}_{u}+\vec{S}_{d}\right)\right\rangle\left(s_{u}+s_{d}\right)_{z}}{\frac{1}{2}\left(\frac{1}{2}+1\right)} \\
& =\frac{\left\langle\left(\vec{S}_{u}-\vec{S}_{d}\right) \cdot\left(\vec{S}_{u}+\vec{S}_{d}\right)\right\rangle \frac{1}{2}}{\frac{3}{4}} \\
& =\frac{2}{3}\left\langle s_{u}^{2}-s_{d}^{2}\right\rangle \\
& =\frac{2}{3}\left[s_{u}\left(s_{u}+1\right)-s_{d}\left(s_{d}+1\right)\right]
\end{aligned}
$$


Since there is only one $\operatorname{spln} \frac{1}{2} \mathrm{~d}$ quark in a proton, $\mathrm{S}_{\mathrm{d}}=\frac{1}{2}$. But the two spin $\frac{1}{2}<$ quarks in a proton can be coupled either to $s_{u}=0$ or $s_{u}=1$. ordinary

Fermi statistics would suggest $S_{u}=0$. But the querk model wave function used Is chosen for other reasons to have $S_{U}=1$. For this wave function

$$
\left\langle\left(s_{u}-s_{d}\right)\right\rangle=\frac{2}{3}\left[2-\frac{3}{4}\right]=\frac{5}{6}
$$

while for $\mathrm{S}_{\mathrm{u}}=0$

$$
\left\langle\left(S_{u}-S_{d}\right)_{z}\right\rangle=\frac{2}{3}\left[-\frac{3}{4}\right]=-\frac{1}{2} .
$$

With this final piece of information, we can calculate the numerical value of the ratio of the proton magnetic moment to the neutron magnetic moment. For the $\mathrm{S}_{\mathbf{u}}=1$ wave function

$$
\begin{aligned}
& \frac{\mu_{p}}{\mu_{n}}=\frac{\frac{1}{4}+\frac{3}{2}\left(\frac{5}{6}\right)}{\frac{1}{4}-\frac{3}{2}\left(\frac{5}{6}\right)} \\
& \frac{\mu_{p}}{\mu_{n}}=-\frac{3}{2}=-1.50
\end{aligned}
$$

Experimental value

$$
\frac{\mu_{p}}{\mu_{n}} \cong-1.46
$$

The $s_{u}=0$ wave function would give $\frac{\mu_{p}}{\|_{n}}=-\frac{1}{2}$ in strong disagreement with experiment. So we see reasonably good agreement between the experimental result and a constituent quark plcture, (with quarks however that seem to Isolate Fermi statistics). 
But why did it take so long to notice that such a construction could be made? (The $\mu_{p}$ and $\mu_{n}$ were found quite early, they appeared in Bethe's book Elementary Nuclear Theory, copyright 1947!). The reason that no one noticed this is that no one would think to take the ratio of $\mu_{p}$ and $\mu_{n}$. Originally, $\mu_{\mathrm{n}}$ and $\mu_{\mathrm{p}}$ were decomposed as

$$
\underset{p}{\mu_{n}}=\mu_{\text {Dirac }}+f_{n}(g)
$$

$f(g)$ is the anomalous moment due to the strong interaction and is a function of the strong interaction coup.ing constant g. Originally, physicists took ratios of the $f(g)^{\prime} s$, hoping to learn about the strong interaction.

Let us review what we have accomplished at this point. By introducirg a constituent quark picture of the proton and neutron, we have been able to predict the ratio of the magnetic moments in a reasonably simple and straightforward way. But in achieving this we have also lost something too. We have been forced to introduce strange new fundamental particles with fractional charges and peculfar statistics. At this point, it is interesting to compare our situation with a plcture of nuclear forces. This comparison w11 lead us to requiring color as a new "charge".

Let us look at nuclear forces in a slightly different way. Imagine that we only know of Even $A, I=0$ nuclei. We would, in this case, assume that the deuteron is a fundamental particle. However, experiments Indicated a structure for the deuteron. We might propose it to be a composite particle, being composed of two new and strange particles that are more fundamental and are called nucleons. Initially, we would be in a quandary though. Since the charge of the deuteron is tle, we would naturally suppose that the charge of the nucleon is $+\frac{1}{2} \mathrm{e}$. The quandary arises when we try to construct the 
deuteron out of two Identical nucleons with $+\frac{1}{2}$ e $\ln$ a ${ }^{1} \mathrm{~S}_{0}$ state. Symmetry considerations forbid this for identical fermions. So the nucleon appears to have fractional charge and peculiar statistics.

But we might postulate that there 1s, In fact, a new degree of freedom such that there are two nucleons with the property

New Degree of Freedom red blue
Label

$$
\mathrm{P}
$$

n
Charge

tle

Oe

There is an SU(2) "color" symmetry, if the deuteron is a "color singlet", antisymmetric in color, it must be symmetric in space and spin. This solves the statistics problea. This "color" of course is what we call isospin or flavor in nuclear physics.

Let us now consider quarks with three colors in addition to the space, spin and isospin degrees of freedom.

By requiring the three quarks in the nucleon to be "color singlet" In the SU(3) color group, we require total symmetry of space, spin S, and total isospin I, we get

$L=0$ color singlets (from symmetry considerations)

$$
\begin{array}{lll}
I=\frac{1}{2} & S=\frac{1}{2} & \text { Nucleon } \\
I=\frac{3}{2} & S=\frac{3}{2} & \Delta
\end{array}
$$


baryons are composed of 3 quarks. We assume effective two-body interactions between the quarks. With this assumption, a simple she11 model mass formula may be written,

$$
m(h)=\sum_{i=1} m_{i}+\sum_{1>j} \frac{\dot{\sigma}_{1} \cdot \dot{\sigma} .}{f_{1 j}} u_{1 j}+v_{1 i}+\ldots
$$

where

$$
\begin{array}{ll}
m_{f} & \text { quark masses } \\
\sigma_{i} & \text { spin mat:ices } \\
U_{i j} & \text { interaction potential } \\
V_{i j} & \text { inceraction potential } \\
f_{1 j} & \text { flavor dependent parameter }
\end{array}
$$

We consider the flavor dependence and spin dependence of baryon and meson masses

\subsection{Flavor dependence}

Let us begin by considering the mass differences chosen to cancel the configuration of the spin-dependent term. For baryons, we obtain

$$
M_{\Lambda}-M_{N}=177 \mathrm{MeV}=m_{s}-m_{u}
$$

where

$\begin{array}{llll}M_{\Lambda} & \Lambda \text { mass } & m_{s} & \text { strange quark mass } \\ M_{N} & \text { Nucleon mass } & m_{u} & \text { up quark mass }\end{array}$


Similarly for mesons

$$
\frac{3}{4}\left[M_{k} \star-M_{\rho}\right]+\left[M_{K}-M_{\pi}\right]=m_{s}-m_{u}=180 \mathrm{MeV}
$$

where

\begin{tabular}{|c|c|c|}
\hline$M_{K} *$ & $K^{\star}$ & mass \\
\hline$M_{\rho}$ & $\rho$ & mass \\
\hline $\mathrm{M}_{\mathrm{K}}$ & K & mass \\
\hline$M_{\pi}$ & $\pi$ & mass \\
\hline
\end{tabular}

The difference in mass between the strange quark and the up quark is essentially the same in both baryons and mesons.

\subsection{Spin dependence}

Now let us look at additional baryon systems. For the systems $\Sigma^{+}$ and $\Sigma^{*+}, S=1 / 2, S=3 / 2$

$$
M\left(\Sigma^{*+}\right)-M\left(\Sigma^{+}\right)=\frac{3}{2}\left(v_{1}^{u s}-v_{0}^{u s}\right)
$$

where $\mathrm{V}_{S}^{\mathrm{ab}}$ denotes the interaction between two quarks with flavors $a b$ and in the state of spin $S$. In this mass difference, the only spin dependent terms result from the u-s interactions in different spin states.

$$
\begin{aligned}
& \text { Similarly, for the } \equiv, \equiv^{*} \text { system } \\
& M\left(\equiv^{\star}\right)-M(\equiv)=\frac{3}{2}\left(v_{1}^{\text {us }}-v_{0}^{\text {us }}\right)=M\left(\Sigma^{*}\right)-M(\Sigma)
\end{aligned}
$$

these systems lead us to consider the $\Delta N$ system again 


$$
\begin{aligned}
M(\Delta)-M(N) & =\frac{3}{2}\left(V_{1}^{u d}-V_{2}^{u d}\right) \\
& =\frac{3}{2}\left(2 M\left(\Sigma^{\star}\right)+M\left(\Sigma^{*}\right)-3 M(\Lambda)\right) \\
307 \mathrm{MeV} & \simeq 294 \mathrm{MeV}
\end{aligned}
$$

so a constituent quark picture gives reasonable results. We might also compare ratios for baryons

$$
\frac{M_{\Delta}-M_{N}}{M_{\Sigma}-M_{\Sigma}}=\frac{f_{\text {su }}}{f_{u u}}=1.53
$$

and for mesons

$$
\frac{M_{P}-M_{\pi}}{M_{K}{ }^{*}-M_{K}}=\frac{f_{\text {su }}}{E_{u u}}=1.61
$$

The consistency of these results leads us to belleve that the constituent quark model applies to baryons and mesons alike.

\section{Additional Evidence of Substructure ... the Antiproton}

So far we have seen that the constituent quark picture, using a shell model, glves good agreement with known masses and magnetic moments. The antiproton also gives us some evidence of substructure. The relevant question to be asked is, "What would we see?" In p $\bar{p}$ annihilation, we see a mean multiplicity of about $5 \pi^{\prime} \mathrm{s}$. However, if $\overline{\mathrm{p}}$ is elementary, the statistical multiplicity of $\pi^{\prime} s$ is between 2 and 3 , rather than the observed value of 5 . What is happening here - the $\bar{p}$ has a substructure of quarks. Furthermore, if the $\bar{p}$ is fundamental, $p \bar{p}$ annihilation should produce a virtual photon and then 
$e^{+} e^{-}$palrs of a rate given by QED calculations for point particles. But this is not observed. He observe plons. And why aga1n, so many pions?

A simple explanation of the number of plons in $p \bar{p}$ annihilation experiments comes from the constituent quark model of the $p$ and the $\bar{p}$. The quarks simply rearrange themselves, and a simple "back of the envelope" calculation gives a multiplicity of 5.25 plons, which agrees roughly with experiment.

\subsection{Quark rearrangement}

Begin by assuming, as before, that the proton and now the $\vec{p}$ are composed of 3 quarks, and 3 antiquarks respectively. The quarks will rearrange themselves in the following manner

$$
3 q+3 \bar{q}+3 \text { mesons }
$$

The $q \vec{q}$ is efther fn a singlet or triplet state. There is a 3:1 statistical favoring of the triplet state (vector meson) over the singlet state (pseudoscalar meson). He neglect isoscalar mesons, and assume that the pseudoscalar meson is always a plon, and the vector meson is always a $\rho$, which gives an extra pion when 1 decays. So we always get at least 3 plons from annihilation, and we get an additional pion from every $\rho$ meson produced. Now we can work through the numbers.

The probability of producing a over the $\pi$ shows the statistical favoring

$$
\begin{array}{ll}
p(\rho)=\frac{3}{4} & \text { probability for a } \rho \\
p(\pi)=\frac{1}{4} & \text { probability for a } r
\end{array}
$$


now consider the following cases: The $3 \pi$ decay gives no additional pions. The probability is given by

$$
p(\pi \pi)=\left(\frac{1}{4}\right)^{3}=\frac{1}{64} \quad 3 \text { pions }
$$

The $\rho \pi \pi$ case gives one auditional $\pi$. The prabability of this case is

$$
p(\rho \pi \pi)=3\left(\frac{3}{4}\right)\left(\frac{1}{4}\right)^{2}=\frac{9}{64} \quad 4 \text { pions }
$$

for the case of $p p \pi$, we get 2 additional pions. The probability is

$$
p(\rho \rho \pi)=3\left(\frac{3}{4}\right)^{2}\left(\frac{1}{4}\right)=3 \times \frac{9}{64} \quad 5 \text { pions }
$$

and finally, we might get $\rho \rho \rho$. This produces 3 additional pions. The probability of producing $p p p$ is

$$
p(\rho \rho \rho)=\left(\frac{3}{4}\right)^{3}=\frac{27}{64} \quad 6 \text { pions }
$$

the mean multiplicity in this simple model is given by

$$
\text { \# plons }=\sum P_{1} \mathrm{n}_{1} \quad \text { sum over all the above decay modes }
$$

where

$$
\begin{array}{ll}
P_{1} & \text { probability of a particular mode } \\
n_{1} & \text { the number of resultant pions }
\end{array}
$$

so

$$
\text { \#pions }=\left(\frac{1}{64}\right) \times 3+\left(\frac{9}{64}\right) \times 4+\left(\frac{27}{64}\right) \times 5+\left(\frac{27}{64}\right) \times 6=5.25 \text { pions. }
$$




\subsection{The Fermi-Yang-Sakata mode1}

Another result of $\mathrm{p} \overline{\mathrm{p}}$ annihilation was the demise of the now forgotten Fermi-Yang-Sakata mode1. A natural model with SU(3) symmetry was the old Fermi-Yang model of the pion as a nucleon, antinucleon pair, extended by Sakata to the strange particles by including the $\Lambda$ with the two nucleons as fundamental. Some of the interactions that we might see in the Sakata model are
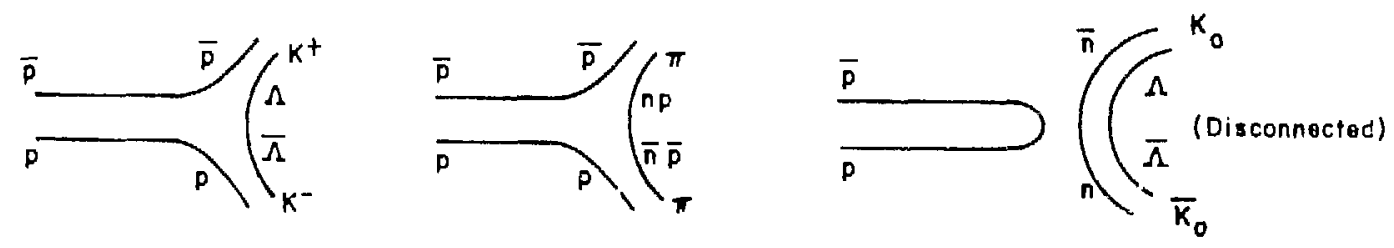

This process is forbidden by unitary symmetry. It should also be strongly suppressed by the OZI rule ... it is a disconnected diagram.

Observation of all three of these processes with equal irequency killed the Fermi-Yang-Sakata model.

\section{Tie Existence of Color}

So far, experimental data has forced us to the constituent quark picture. More data will force us towards QCD, (colored quarks, gluons).

\subsection{The saturation problem}

The problem of saturation is well known to nuclear physicists. Saturation leads us to the existence of colored quarks in the study of quarkquark interactions. 
Take the reaction

$$
\mathrm{d}+{ }^{3} \mathrm{H}+\mathrm{n}+\mathrm{a} \quad \text { galn in energy }
$$

(attractive force of 3 nucleons in ${ }^{3} \mathrm{H}$ on the proton in the deuteron is stronger than the force of the one neutron in the deuteron). So why not

$$
\text { meson + baryon } \rightarrow q+\overline{q q q q}
$$

Why doesn't this reaction occur ilke the aforementioned nuclear reaction? Why aren't two plons bound? How does this work -- if $q q$ and $q \bar{q}$ forces are attractive, why do we only see bound $q \bar{q}$ and $q q q$ systems. Let us begin by considering a naive model.

\subsection{Na1ve model}

In this first naive case, we assume that $q$ and $\bar{q}$ are heavy, and the binding energy cancels the mass

$\begin{array}{llll}\text { meson } & q \bar{q} & 1 \text { interaction } & 2 \text { masses cancelled } \\ \text { baryon } & q q q & 3 \text { interactions } & 3 \text { masses cancelled }\end{array}$

this leads us to

$$
V(q q)=\frac{1}{2} V(q \bar{q})
$$

At this point it is instructive to return to our picture of nuclear physics where the deuteron is a fundamental particle and all nuclei have $S=0$. The question to ask is how do we have deuteron states and get no bound multi- 
nucleon states with I other than 0 ? The answer to this question parallels the explanation of how bound $q q q$ and $q \bar{q}$ systems are related to color.

Let us begin by postulating that the nucleons interact through the exchange of a $p$ vector meson. For identical nucleon, e.g. protons

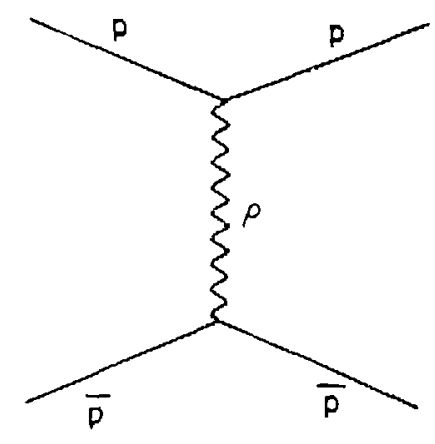

the $\bar{p} \bar{p}$ interaction is attractive

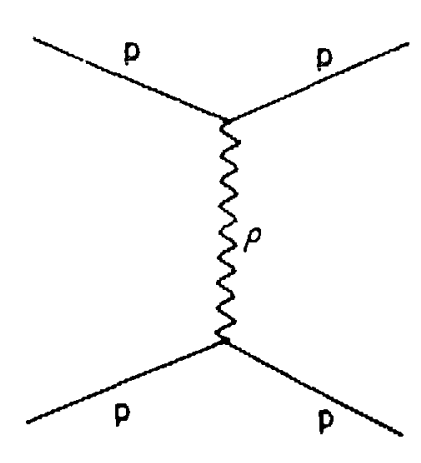

the pp interaction is repulsive

The Interaction that we are considering may be written formally for the general two-nucleon case as

$$
v_{12}=U\left(\sigma_{1}, \sigma_{2}, r_{12}\right)\left(\vec{\tau}_{1} \cdot \dot{t}_{2}\right)
$$

where $\vec{t}$ is isospin, so for

$$
\begin{array}{ll}
I=1 & \stackrel{+}{\tau}_{1} \cdot \stackrel{+}{\tau}_{2}=+1 \\
I=0 & \stackrel{+}{\tau}_{1} \cdot \stackrel{+}{\tau}_{2}=-3
\end{array}
$$

and

$$
\overline{p p}=\frac{1}{\sqrt{2}}(I=1)+\frac{1}{\sqrt{2}}(I=0)
$$


Now consider the $\mathrm{N}$-particle system, where the interaction may be written

where

$$
V=\sum_{1>j}^{N} \vec{T}_{1} \cdot \vec{T}_{j} U_{1 j}\left(a_{1}, a_{j}, r_{i j}\right)
$$

$$
\begin{aligned}
& \overrightarrow{\mathrm{T}}_{1} \quad \text { isospin operator for particle } 1 \\
& U_{1 j} \quad \text { interaction potential. }
\end{aligned}
$$

The expectation value of the interaction $V$ can be mircen as

$$
\begin{aligned}
\langle V\rangle & =\frac{1}{2} \sum_{1, j}\left(\vec{T}_{1} \cdot \overrightarrow{\mathrm{T}}_{j}\right)\langle u\rangle-\sum\left\langle\overrightarrow{\mathrm{T}}_{1} \cdot \overrightarrow{\mathrm{T}}_{1}\right\rangle\langle u\rangle \\
& =\frac{1}{2}\left(\sum_{1} \overrightarrow{\mathrm{T}}_{1} \cdot \sum_{j} \overrightarrow{\mathrm{T}}_{j}\right)\langle u\rangle-\sum\left(\overrightarrow{\mathrm{T}}_{i} \cdot \overrightarrow{\mathrm{T}}_{i}\right\rangle\langle u\rangle \\
& =\frac{1}{2} I(I+1)\langle u\rangle-\mathrm{n} \frac{3}{4}\langle u\rangle
\end{aligned}
$$

where $\vec{I}=\sum_{1} T_{1}$ is the total isospin, $\langle u\rangle$ denotes $\left\langle U_{g}\right\rangle$ assumed to be independent of $u$ and $j$, and $n$ is the number of particles.

The lowest state, $I=0$ gives us the interaction energy of the ground state of the n-particle system

$$
V(n)=n \frac{3}{4}\langle u\rangle \Rightarrow V(2)=\frac{3}{2}\langle u\rangle
$$

$V$ is proportional to $n$, the number of partisles. It is this proportionality to $n$ that is responsible for the saturation of the deuteron. You do not "galn anything" ( 1 n binding energy per particle) by going to larger $\mathrm{I}=0$ systems over $11 / 2$ deuterons. 
So we see that $p p$ and $p \bar{p}$ are both aitractive in the isosinglet ... this leads to isoscalar mesons, the deuteron, and nothing further.

This example ieads us to color and quarks. It shows us that if the system ri: Includes two colors, we only get $q q$ baryons and mesons, with no strongly bound multiquark states. But look at quark-quark interactions in a 3 color system. The quarks interact via the exchange of a vector particle we wlll call a gluon, reminiscent of the nuclear case where two nucleons interact by exchanging $D^{*}$ mesons. In the gluon case though, there are 8 states of the colored gluon (for the $p$ with only 2 colors, there were only 3 states of the mediating particle $\rho^{\circ}$ and charge changing $\rho^{+}$and $\rho^{-}$).

For the three color system, there are 6 color changing gluons (11ke $\rho^{ \pm}$) and 2 non-color changing gluons (like $\rho^{\circ}$ ). Diagrammatically this is

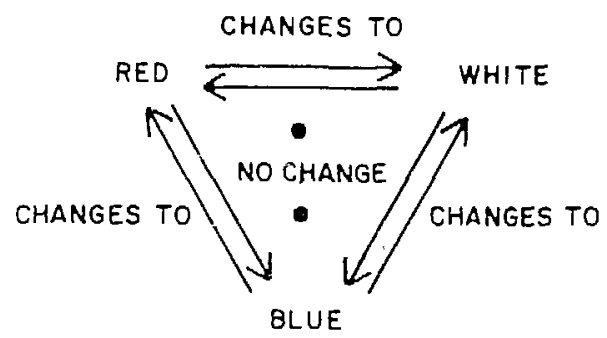

In general, for $n$ colors, there are $n^{2}-1$ gauge bosons. Like in our nuclear physics example, we can write out the interaction as

$$
v_{12}=U\left(\sigma_{1}, \sigma_{2}, r_{12}\right)\left(\vec{F}_{1} \cdot \stackrel{+}{F}_{2}\right)
$$

where $U$ is an interaction and $F$ is the color operator (this is isospin in the two color case we discussed). Then 


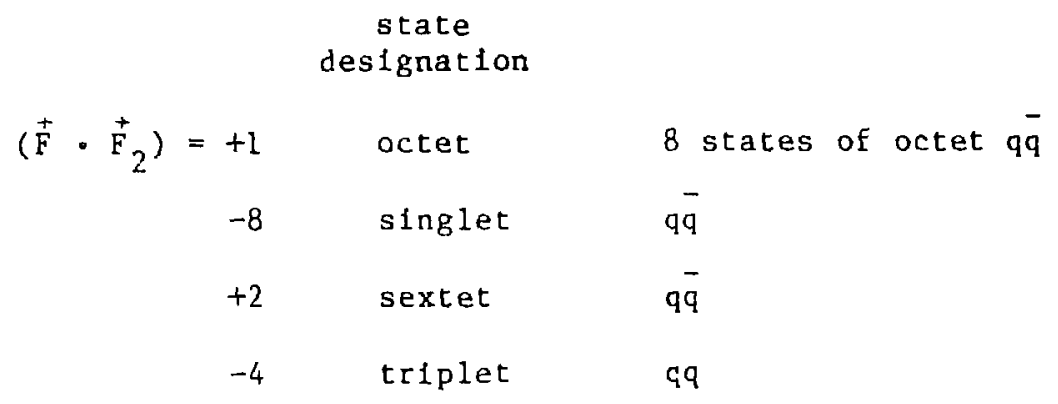

Note that the antisymmetric color triplet state of two quarks has half of the Interaction of the color singlet quark-antlquark system, again giving V(qq) $1 / 2 \mathrm{~V}(\mathrm{qq})$. The designation of the 3 color system reduces to singlet and triplet in the 2 color system. Following our previous example, we rewrite $V$

$$
\begin{aligned}
\langle V\rangle & =\frac{1}{2}\left(\sum_{i} F_{1} \cdot \sum_{j} F_{j}\right)\langle u\rangle-\sum_{i} F_{1} \cdot F_{1}\langle u\rangle \\
& =\frac{1}{2} c\langle u\rangle-n c\langle u\rangle
\end{aligned}
$$

$C$ is the Casimir operator, whose minimum is 0 in the singlet state.

When you have made singlet states, states other than $3 q$, baryons and $q \bar{q}$ mesons gain no blnding energy. It takes no energy to pull larger systems apart into the $3 q$ and $q \bar{q}$ singlet states. We observe larger nultiquark systems (nuclei) but they result from much smaller and finer effects.

\subsection{Masses of baryons and mesons}

The mass of the baryons and mesons in the 3 color constituent quark model can now be calculated. We begin with the baryons. The Hamiltonian is

$$
\mathrm{H}_{\mathrm{B}}=\mathrm{T}_{1}+\mathrm{T}_{2}+\mathrm{T}_{3}+\mathrm{V}_{12}+\mathrm{V}_{13}+\mathrm{V}_{23}+3 \mathrm{M}
$$


I is the kinetic energy, $V$ is the quark interaction and $M$ is the quark mass. For the mesons

$$
H_{m}=T_{r e 1}+2 V_{12}=T_{1}+T_{2}+2 V_{12}+2 M-\frac{\left(p_{1}+p_{2}\right)^{2}}{4 M}
$$

where we have used the result $V(q q)=\frac{1}{2} V(q \bar{q})$, and subtracted the kinetic energy of center of mass motion. Make the ansatz that the exact solution of the baryon wave function is known and is given by $\psi_{B}$. That is ...

$$
\left\langle\psi_{B}|H| \psi_{B}\right\rangle=3\left\langle T_{1}\right\rangle+3 M+3\left\langle V_{1 j}\right\rangle=M_{B}
$$

$M_{B}$ is baryon mass

Using this solution as a trial wave function for mesons using the variational principle

$$
\begin{aligned}
& M_{m}<\left\langle J_{B}\left|H_{m}\right| \psi_{B}\right\rangle=2\left\langle T_{1}\right\rangle+2\left\langle V_{1 j}\right\rangle+2 M-\frac{\left\langle T_{i}\right\rangle_{B}}{2} \\
& M_{m}<\frac{2}{3} M_{B}-\frac{\langle T\rangle_{B}}{6}
\end{aligned}
$$

To estimate $\langle\mathrm{T}\rangle_{\mathrm{B}}$ we use the virial the srem and the simple logarithimic potential used to fit the experimental charmonium spectrum

$$
\begin{array}{ll}
2 v_{1 j}=U_{0} \ln \left(r / r_{0}\right) & U_{0}=-733 \mathrm{MeV} \\
\frac{\left\langle T_{B}\right\rangle}{6}=\frac{1}{6} U_{0} \cdot \frac{3}{4}=\frac{U_{0}}{8}=-90 \mathrm{MeV} &
\end{array}
$$


To compare these results with experiment we use spin-averaged meson and baryon masses

$$
\begin{aligned}
& M_{B}=\frac{1}{2}\left[M_{N}+M_{\Delta}\right]=1085 \mathrm{MeV} \\
& M_{m}=\frac{3}{4} M_{\rho}+\frac{1}{4} M_{\pi}=613 \mathrm{MeV}
\end{aligned}
$$

the predicted meson mass is

$$
M_{\mathrm{D}}<\frac{2}{3} M_{B}-\frac{\left\langle T_{B}\right\rangle}{6}=633 \mathrm{MeV}
$$

The difference in the two values is approximately $20 \mathrm{MeV}$ and the inequality is In the right direction. The mass calculated from the colored quark plcture is of the right order of magnitude. We see also that the effective masses of the quarks in baryons and mesons are different.

A better approximation for the mass is obtained if we allow the sizes of the baryons and mesons to be different. This is known as a scale approximation. We st 111 take

$$
M_{m} \cong\left\langle\psi_{B}(K r)\left|H_{m}\right| \psi_{B}(K r)\right\rangle
$$

but now we include a scale parameter $k$ chosen to minimize $\left\langle\mathrm{H}_{\mathrm{m}}\right\rangle$. Using the same method as before, with the same potential, but including a size dependent parameter, we obtain

$$
M_{\text {m }} \cong \frac{2}{3} M_{B}-106=617 \mathrm{M} \in \mathrm{V}
$$


Begin by considering states composed of q $\bar{q}$ pairs (the mesons)

$$
|\phi\rangle=\frac{1}{\sqrt{3}}\{|r \bar{r}\rangle+|b \bar{b}\rangle+|g \bar{g}\rangle\}
$$

$r, b, g$ are the three colors. For a general number of colors

$$
|\psi\rangle=\frac{1}{\sqrt{N}} \sum_{c}^{N}\left|q_{c} \bar{q}_{c}\right\rangle
$$

where $[1$ s the sum over $N$ colors. We could now study how the number of colors affects various physical quantitles. Conclder a coior changing interaction. A simple example is a one-gluon-exchange between $q$ and $\vec{q}$ pairs

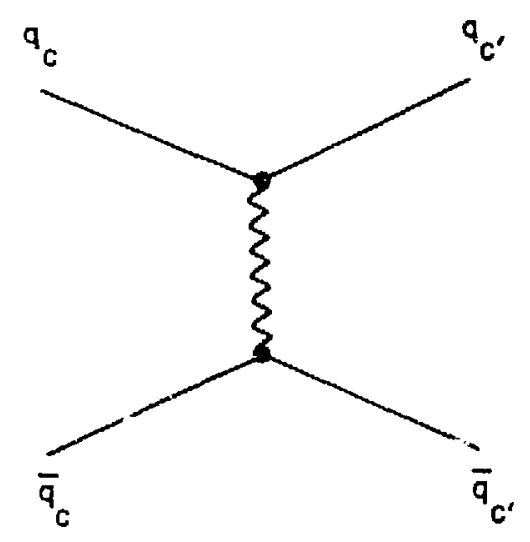

The matilx element for this process is

$$
\left\langle\psi_{a}\left|g^{2} U\right| \psi_{b}\right\rangle=\frac{g^{2}}{N} \sum\left\langle q_{c} \bar{q}_{c}|U| q_{c} \bar{i}_{c}{ }^{\prime}\right\rangle
$$

Since U Is a color changing operator (gluon exchange) the sum ylelds $\mathrm{N}^{2}$ terms

$$
\left\langle\psi_{a}\left|g^{2} U\right| \psi_{b}\right\rangle=g^{2} N\left\langle q_{c} \bar{q}_{c}|U| q_{c}, \bar{q}_{c}\right\rangle
$$


This result does depend on the number of colors. If we are interested in meson-meson scattering, we need to calculate

$$
\left.\left\langle q_{c} \bar{q}_{c} \cdot\left|g^{2} u\right| q_{c} \bar{q}_{c}\right\rangle^{\prime}\right\rangle=g^{2}\left\langle q_{c} \bar{q}_{c},|u| q_{c} \bar{q}_{c}{ }^{\prime}\right\rangle
$$

To do this problem we will use an expansion for large $N$ (the large $N$ limft) such that

$$
\begin{aligned}
& \mathrm{g}+0 \text { but } \mathrm{g}^{2} \mathrm{~N} \text { is fixed } \\
& \mathrm{N}+\infty
\end{aligned}
$$

(note: Modern Critical Phenomena by Ma contains a nice discussion on how $1 / \mathrm{N}$ expansions work)

$$
\begin{array}{ll}
\mathrm{g}^{2} \mathrm{~N} & \text { is fixed for bound mesons } \\
\mathrm{g}^{2} & +0 \quad \text { leads to noninteracting mesons }
\end{array}
$$

This large $N$ limit was first presented in the Les Houches Lectures (no refereed journal would take it at the time!). Since then it has been "rediscovered" for work in QCD where it is used to make $1 / \mathrm{N}$ expansions to calculate qualitative features.

But how well does all of this work for baryons? It turns out that the properties of baryons do not come out easily, and care must be taken in the large $\mathrm{N}$ 1imit. There has been a resurgence of soliton solutions, as well as pion fields, as descriptions of baryons. We must study how these kinds of approaches to baryons preserve the 1dea that baryons and mesons are made of the same stuff.

But let us get back to our initial task of looking at observables that depend on color or the number of colors. While we have demonstrated that 
quantities may be dependent on the number of colors, we have not seen how to extract $N$ from the observables considered. We will study this now, beginning with the: process of annihilation. Consider

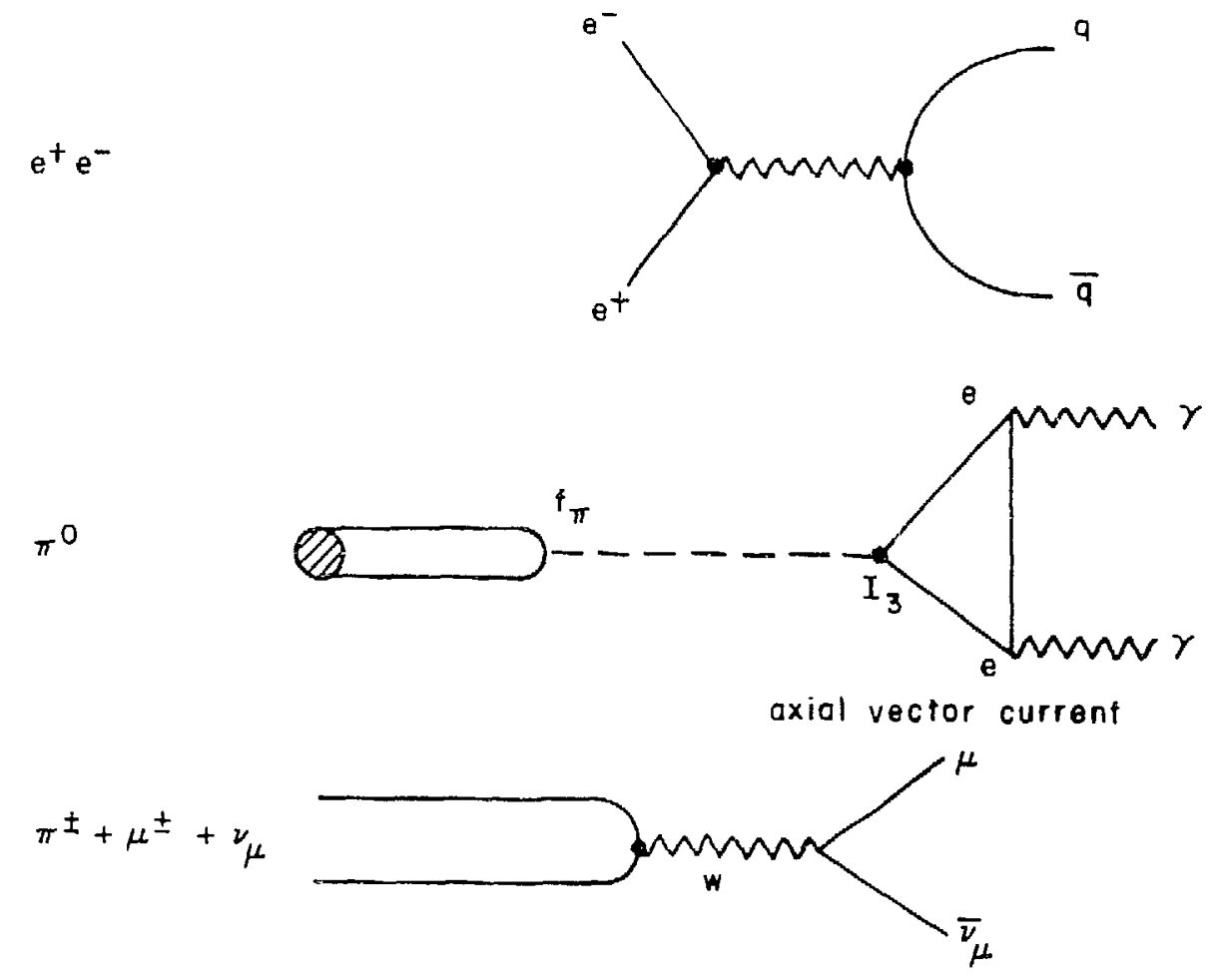

To consider these processes, we need to calculate

$$
\langle\psi|\mathrm{J}| 0\rangle \text { (color Independent) }=\frac{1}{\sqrt{N}} \int_{c_{\text {(meson) }}}^{\left\langle q_{c} \bar{q}_{c}|J| 0\right\rangle}
$$

which becomes

$$
=\sqrt{N}\left\langle q_{c} \bar{q}_{c}|J| 0\right\rangle
$$

If $J$ is a color singlet operator that changes the number of quark factors involving the number of colors appear. The decay width is proportional to the 
square of the matrix element.

$\left(\sqrt{N}\left\langle q_{c} \bar{q}_{c}|J| 0\right\rangle\right)^{2}$

thus the decay width gives a way to find $N$ experimentally if we can calculr.

$$
\left\langle\mathrm{q}_{\mathrm{c}} \overline{\mathrm{q}}_{\mathrm{c}}|\mathrm{J}| 0\right\rangle
$$

For $\pi^{0}$ decay, this marrix element is calculated from the triangle diagram

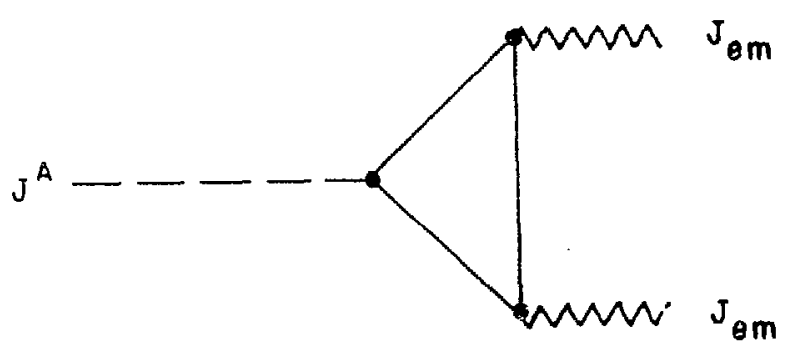

First recall that

$$
\left|\pi^{0}\right\rangle=\frac{1}{\sqrt{2}}[\bar{u}-d \bar{d}]
$$

with this we calculate the trlangle diagram

$$
\begin{aligned}
& \left\langle 0\left|\mathrm{~J}_{\omega}\right| \pi\right\rangle\left\langle\pi\left|\mathrm{J}_{\text {em }} \mathrm{J}_{\mathrm{em}}\right| O\right\rangle=N\left[Q_{u}^{2}-Q_{\mathrm{d}}^{2}\right] \\
& \mathrm{J}_{\omega} \text { operator is for } \pi+\mu U \\
& \mathrm{~J}_{\mathrm{em}} \mathrm{J}_{\text {em }} \text { operators for } \pi+Y Y \\
& N \text { is the number of colors }
\end{aligned}
$$


The rate goes as the matrix element squared, so production rates go as $\mathrm{N}^{2}$. The $\mathrm{e}^{+} \mathrm{e}^{-}$reaction rate does as $\mathrm{N}$ (matrix element is proportional to $\sqrt{\mathrm{N}}$ ). Using this, we can construct the ratio of rates

$$
\text { Ratio }=\frac{\sigma\left(e^{+} e^{-}+x\right)}{\sigma\left(e^{+} e^{-}+\mu^{+} \mu^{-}\right)}=\sum Q^{2} \mathrm{~N}
$$

This gives us a second example that exposes $N$. Once we know $Q_{u}$ and $Q_{d}$, we can see the color factors. In the first example, $\pi^{0}$ decay, we can see

$$
\left\langle 0\left|J_{\omega}\right| \pi\right\rangle\left\langle\pi\left|J_{\text {em }{ }_{\text {em }}}\right| O\right\rangle=N\left[Q_{u}^{2}-Q_{d}^{2}\right]=N\left[\left(Q_{u}+Q_{d}\right)\left(Q_{u}-Q_{d}\right)\right]
$$

but by isospin $Q_{u}-Q_{d}=Q_{p}-Q_{n}$ where $Q_{p}$ is the proton charge, $Q_{n}$ is the neutron charge. Furthermore, the deuteron can be said to have $2 \mathrm{~N}$ quarks $\mathrm{N}$ up quarks and $\mathrm{N}$ down quarks. Using this, we can see that

$$
N\left(Q_{u}+Q_{d}\right)=N Q_{u}+N Q_{d}=Q_{\text {deut }}
$$

which allows us to write

$$
N\left[\left(Q_{u}+Q_{d}\right)\left(Q_{u}-Q_{d}\right)\right]=Q_{\text {deut }}\left(Q_{p}-Q_{n}\right)
$$

$Q_{\text {deut }}, Q_{p}$ and $Q_{n}$ are independent of $N$. If $N$ were one, $p$ and $n$ are fundamental - they are quarks. In this hypothetical case, the trlangle diagram becomes

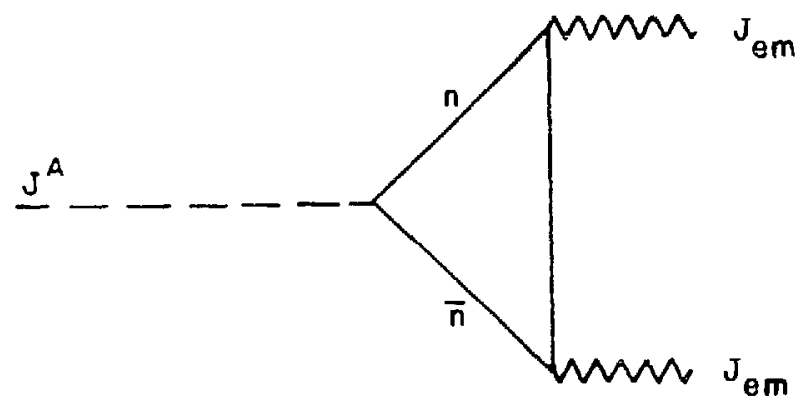


One other color dependent quantity is the one gluon exchange description of a meson decay into two mesons

$$
\begin{aligned}
& \left\langle\psi_{1}\left|\mathrm{~g}^{2} \mathrm{U}\right| \psi_{2} \psi_{3}\right\rangle \quad \psi_{1} \text { is a color singlet state } \\
& \psi_{2}, \psi_{3} \text { are color singlets } \\
& =\frac{1}{N^{3 / 4}} \sum\left\langle q_{c} \bar{q}_{c}\left|g^{2} U\right| q_{c}, \bar{q}_{c}, q_{c} \cdot \bar{q}_{c}{ }^{\prime}\right\rangle
\end{aligned}
$$

Feynman diagram

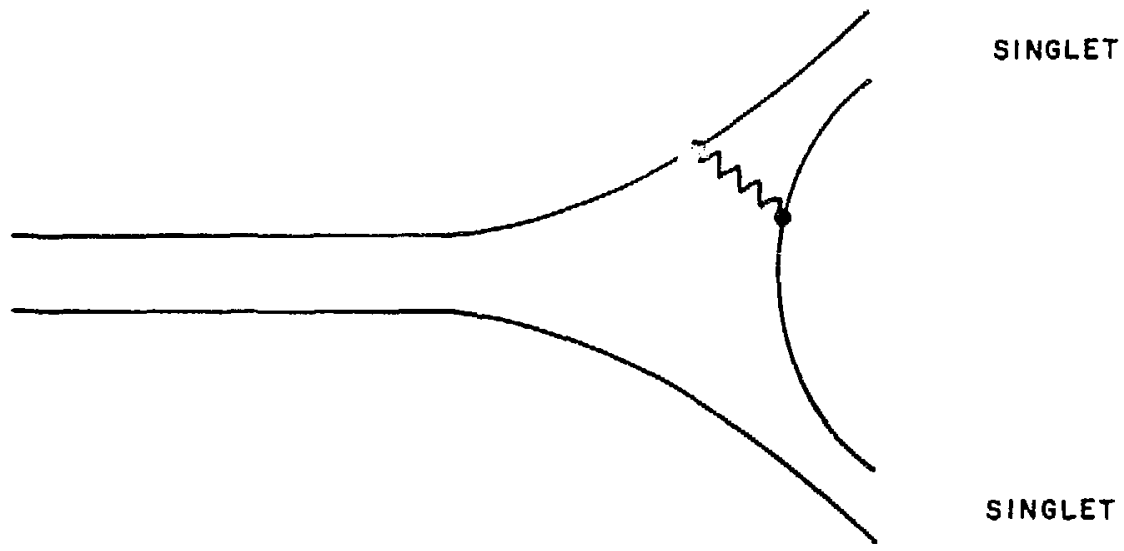

For the case $c=c^{\prime}=c^{\prime \prime}$

$$
\left\langle\psi_{1}\left|g^{2} u\right| \psi_{2} \psi_{3}\right\rangle=\frac{1}{\sqrt{N}}\left\langle q_{c} q_{c}\left|g^{2} u\right| q_{c} \bar{q}_{c} q_{c} \bar{q}_{c}\right\rangle
$$

The number of colors serve to supress the one gluon exchange. Baryons show similar color dependencles. They can be viewed in the following way

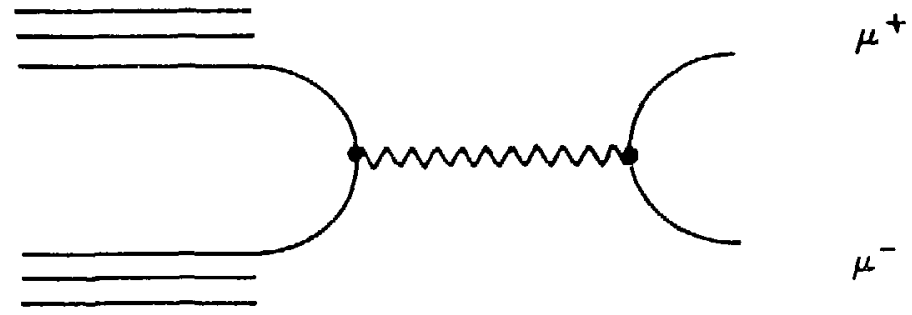


In order to annihilate, a $q$ must find a $\bar{q}$ of the same color. In a baryon, this probability of this occurring goes as $1 / \mathrm{N}$.

6. Hadron Masses and the Hyperfine Interaction

Let us take another look at calculating hadron masses.

Specifically, we look at the "hyperfine" Interactions

$$
s=3 / 2 \quad \begin{array}{ll}
M_{\Delta}-M_{N} \\
\\
M_{\rho}-M_{\pi}
\end{array}
$$

The differences between these different spin particles result from terms that resemble the hyperfine interaction in atomic physics

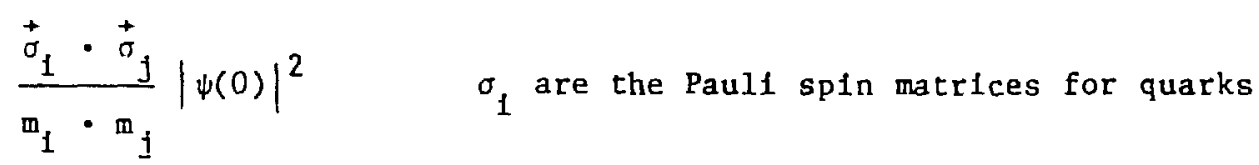

use the reduced wave function

$$
U=r \psi
$$

and the Schroedinger equation

$$
\frac{\partial^{2} U}{\partial r^{2}}=v \frac{\partial U}{\partial r} \quad v \text { is the effective quark-quark interaction }
$$

11ke in atomic physics

$$
\frac{\left|\psi_{1 j}(0)\right|^{2}}{u_{i j}}=K\left\langle\left|\frac{\partial v_{1 j}}{\partial r_{i j}}\right|\right\rangle
$$


in this case, choose a $V$ that confines

$$
\begin{aligned}
& V=K_{c} r \\
& \frac{\partial V}{\partial r}=K_{c}
\end{aligned}
$$

this allows us to make an evaluation without knowing the wave functions.

Using all of these ideas allows us to construct the hyperfine interaction

$$
\begin{aligned}
& \left.\left\langle V_{H P}\right\rangle=\frac{K_{c} v\left\langle\vec{\sigma}_{i} \cdot \dot{\sigma}_{j}\right\rangle}{m_{i}+m_{j}} \quad \frac{\left|\psi_{1 j}(0)\right|^{2}}{\left(\frac{m_{i} m_{j}}{m_{i}+m_{j}}\right)}\right] \\
& =\frac{k_{c} V\left\langle\vec{\sigma}_{1} \cdot \stackrel{\sigma}{\sigma}_{j}\right.}{m_{1}+m_{j}}\left[\frac{\left|\Psi_{1 j}(0)\right|^{2}}{m_{1 j}}\right]
\end{aligned}
$$

where $\mathbb{m}_{1 j}$ is the reduced mass. For the potential that we are considering, this gives, to a good approximation

$$
M_{P}^{2}-M_{\pi}^{2}=M_{K}^{2}-M_{K}^{2}=M_{D}^{2} *-M_{D}^{2}
$$

we recall that (from considerations in atomic phystes)

$$
\frac{\left|\psi_{1 j}(0)\right|^{2}}{m_{1 j}}=C K_{c}\left\langle\frac{\partial \omega}{\partial r_{1 j}}\right\rangle
$$

where $w$ is the potential. This leads us to the following expression for mass splitting due to the hyperfine interaction in baryons,

$$
\frac{\left|\psi_{1 j}(0)\right|^{2}}{m_{1 j}}=C K_{c}\left\{\frac{\partial \omega_{12}}{\partial r_{12}}+\frac{1}{2}\left\langle\cos \theta_{2} \frac{\partial \omega_{23}}{\partial r_{23}}+\cos \theta_{1} \frac{\partial \omega_{13}}{\partial r_{13}}\right\rangle\right\}
$$




\section{More Exot1c Quark Systems}

By taking a nuclear physics approach to the substructure of a nucleon, we get surprisingly good agreement. Use of the color degree of freedom refines these results.

Because of the success of this approach, it is reasonable to continue in the same way when studying more exotic quark states.

We approach these problems using the deuteron model described before. Th1s is the classic approach used by Yang and M11ls, where 1sosp1n 1s like color, let us begin with a few comments on 1sospin.

Isospin symetry is broken by the electromagnetic interaction. If the 1sospin symmetry was not a broken symmetry, there would be no way of discerning protons from neutrons ... they would be degenerate states. Yang and Mills took these considerations of symmetry further. Begin by considering a transformation $\phi(x)$ such that all observables remain unchanged under

$$
e^{1 \phi(x)} \psi(x) \quad(a b e 11 a n)
$$

For non-abelian theorles, $\psi(x)$ is a complex, multivariable quantity that transforms non-trivially, (this transformation is known as a local gauge transformation). Take an example, 1sospin and the nuclear wave function

$$
e^{1+\dot{\tau} \cdot \vec{s}} \psi(x)
$$

According to Pauli, IE Isospin is a non-abelian transformation, even if isospin is a good symmetry we can still discern a difference between pp and pn systems. Take a proton and a neutron that we spacially separated. 
The non-abelian transformation operates different at different points in space. However, problems develop. If lsospin is a good symetry, we can define a direction in 1sospin space and call tt p. But how can we connect space and lsospln? There is no way of defining a direction in 1sospln space and saying it is "the same" direction in all of space time.

We also get into trouble when we try to define a singlet state for spactally separated nuclel.

p

n $\bar{p} \quad$ singlet

$\overline{\mathrm{n}} \quad$ singlet

These states cannot ociur at the same time (the propagation time must be greater than zero). What is really golng on is the emission of vector hosons.

$$
\begin{aligned}
& p+n \\
& \bar{p} \rightarrow \bar{n}
\end{aligned}
$$

When we apply a transformation (local gauge transformation) 11ke before, we w111 rotate the fermions and the vector bosons. Th1s leads to problems. A great deal of work has been done on local gauge transformation using lattice gauge theories. Some progress has been made, and QED is an example. Take for example, the charge observed on a particle, QED gIves us the plcture

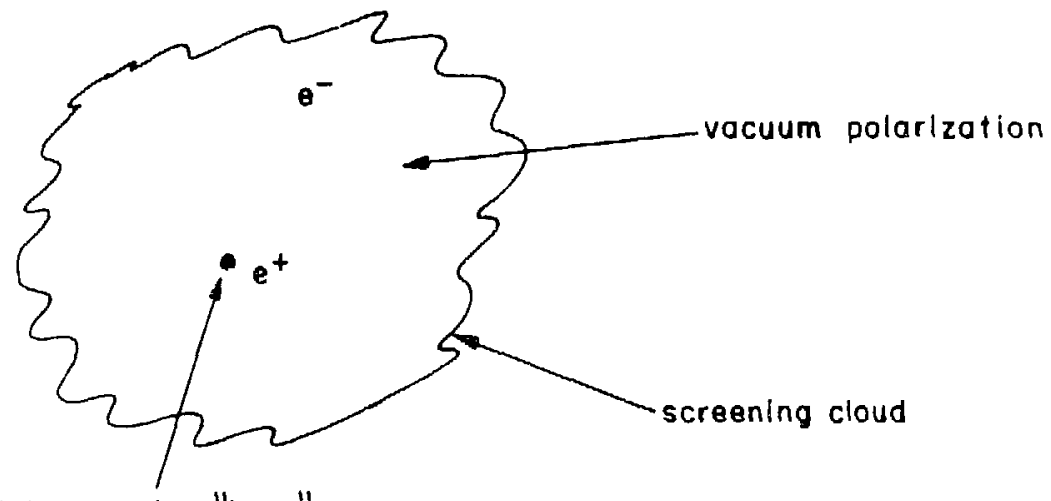

particle with negative "bare" charge 
A particle with some bare charge is surrounded by a screening cloud that results from polarizing the vacuum. Outside the cloud we see that one photon exchange describe the electromagnetic interaction. QED is an abelian theory - the $e^{+}$may emit photons but the photons carry no charge, so they do not screen. QCD is a non-abelian gauge theory. QCD vector bosons (the gluons) carry "charge" so they can act to screen. Furthermore, unl1ke photons, gluons may emit other gluons. A nuclear physlcs example clarffles this point further

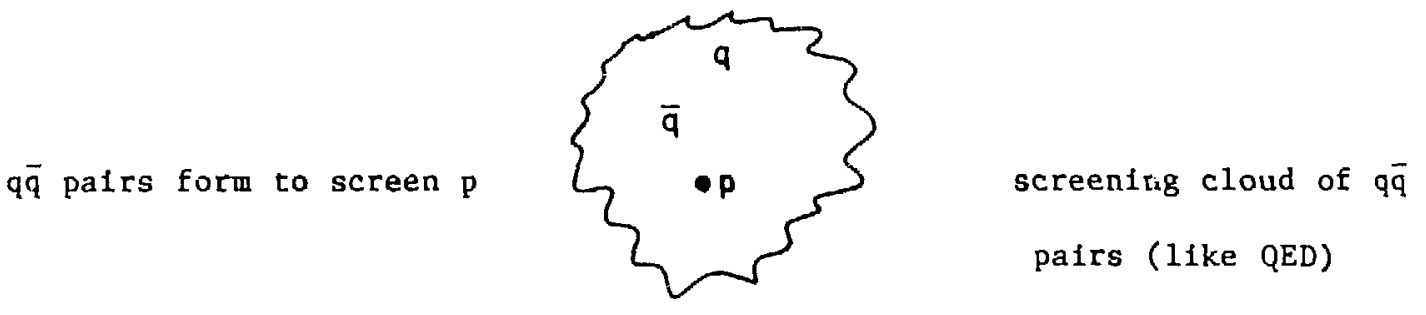

screening

p goes to a $\mathrm{n}^{+}$states, with $p^{+}$forming the cloud.

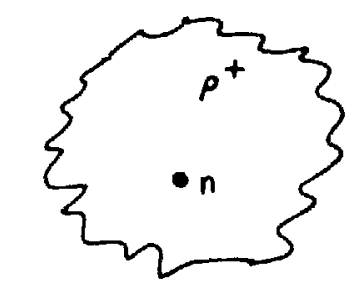
different from QED

anti-screening

Which effect dominates (screening vs anti-screening) depends on the number of quark flavors. If the number of flavors gets large, asymptotic freedom disappears. Using the same example of $n p^{+}$, we can see how confinement might come about. Consider the diagram 


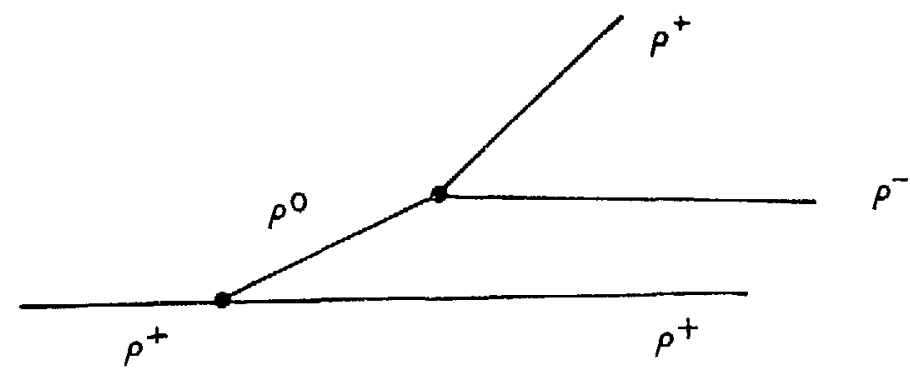

Because of this prolifercation the "force" goes up, so we get confinement. Lattice calculations begin with the philosophy: "we know asymtotic freedom, so ignore short distance effects. Define the field at a number of discreet space time points, and try to obtain long-range effects". For example, place two quarks at the same distance from each other<smiles>[SiH3][SiH3]</smiles>

First, solve the field equations, and then find the interaction, as a function of separation. It turns out that the interaction goes as:
$1 / \mathrm{r}$
short distance
r
long distances

This type of result has been very successful in describing mesons and baryons, but useless for higher number multiquark systems.

Using simple potentials has allowed us to obtain some basic features, (saturation, "neutral" hadrons). The question is how to go further. One might begin by looking at an analogy in molecular physics

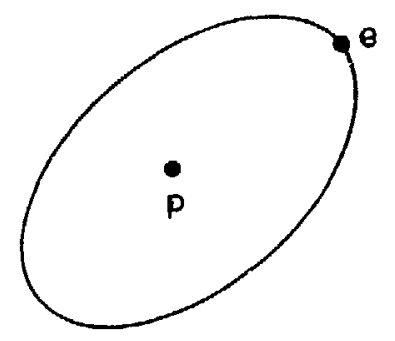


The result from this plcture is a Van der Waals interaction between molecules. Can this plcture describe nucleon-nucleon interactions, or other multiquarkmultiquark phystes?

QCD is different from a dipole fleld. The color fleld is confined In a flux tube - it is not like the molecular case
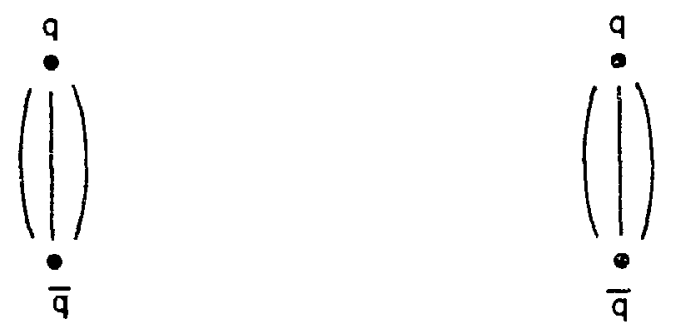

the $q \bar{q}$ pairs must get close together to interact. When the palrs do get clfose we could get a flip

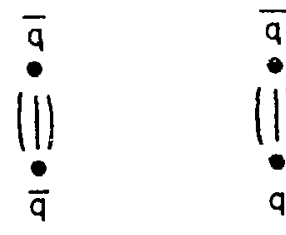

f11p
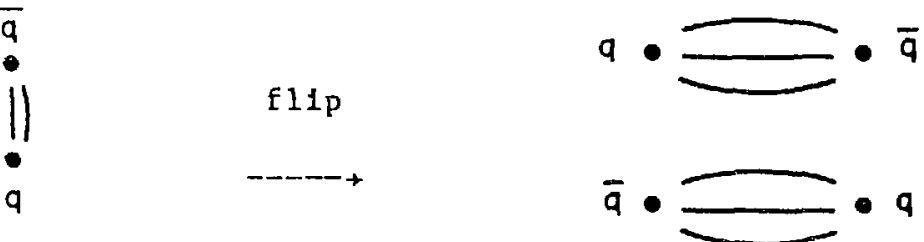

For example $\pi^{+} \pi^{-} \rightarrow \pi^{0} \pi^{0} \quad$ is a case of

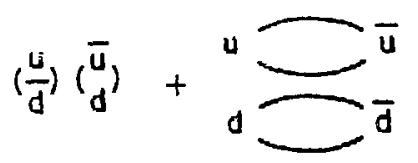

(the states

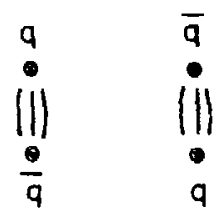

$q \cdot \bar{q}$

and

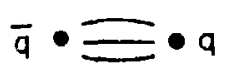




$$
\begin{aligned}
& \psi_{\mathrm{m}}(\mathrm{qq})=x_{\text {color }} \cdot \phi(x, \sigma \ldots) \\
& \psi_{B}(q q q)=x_{\text {color }} \cdot \phi(x \ldots)
\end{aligned}
$$

We cannot do this for more than 3 quarks though. We cannot write an effective potential. For example, take a $4 \mathrm{q}$ system. The wave function of a $4 \mathrm{q}$ system is a $2 \times 2$ matrix in color space. This wave function is not gauge invariant if we transform only the quarks. The requirement of transforming quarks and gluons makes writing an effective interaction difficult. Even a confining potential is unphysical since as $q$ 's are separated, a $q \bar{q}$ pair is formed.

\section{A Study of Multiquark Systems}

So there are many difficulties involved in extrapolating what we know in the $q \bar{q}$ and $q q q$ cases to higher number multiquark systems. There are new effects that we cannot calculate. Now we will try to overcome some of these difficulties to get quantitative or semiquantitative results. First, let us begin by reviewing why multiquark systems are exotic and weakly bound. To do this let us begin with a potential model that is like the 0 exchange model formerly used for the deuteron. The potential is of the form

$$
\begin{aligned}
U= & \sum \lambda_{1} \lambda_{j} U\left(r_{1 j}\right) \\
\langle U\rangle & =\sum_{1\rangle j} \lambda_{1} \cdot \lambda_{j}\left\langle U\left(r_{1 j}\right)\right\rangle \\
& =\frac{1}{2} C(N)-n C(3)
\end{aligned}
$$

where $\lambda$ 's are the operators for color (analogous to $\sigma^{\prime} s$ for angular momentum), $C$ is a constant, $n$ is the number of particles. 
What we obtain from such a potential model is a bindiag energy that Is proportional to the number of particles, so the mass of a hadron is proportional to the number of quarks. This result was first found by Nambu. Nambu supposed that hadrons are bound by one gluon exchange, and arrived at: the hadron mass formula:

$$
\begin{aligned}
& M(H)=n_{0} n_{q}(H) \\
& M(q \bar{q})=2 m_{0}
\end{aligned}
$$$$
\text { Any multiquark system will simply break up into }
$$$$
\text { a system of } 3 q \text { and } q \bar{q} \text { sets. }
$$

Before golng onto look at more multiquark systems, it is useful to review some angular momentum algebra. Recall:

$$
\begin{aligned}
& \mathrm{J}^{2}=\mathrm{J}_{\mathrm{x}}^{2}+\mathrm{J}_{\mathrm{y}}^{2}+\mathrm{J}_{\mathrm{z}}^{2} \\
& \mathrm{~J}^{2} \psi=\mathrm{f}(\mathrm{j}+1) \psi \quad \mathrm{J} \text { is the total angular momentum } \mathrm{J}_{\mathrm{T}}=\mathrm{J}_{1}+\mathrm{J}_{2} \\
& \mathrm{~J}_{1} \cdot \mathrm{J}_{2}=\frac{1}{2}\left[\mathrm{~J}_{\mathrm{T}}^{2}-\mathrm{J}_{1}^{2}-\mathrm{J}_{2}^{2}\right] \\
& =\frac{1}{2}\left[j_{\mathrm{T}}\left(\mathrm{j}_{\mathrm{T}}+1\right)-\mathrm{j}_{1}\left(\mathrm{j}_{1}+1\right)-j_{2}\left(\mathrm{j}_{2}+1\right)\right]
\end{aligned}
$$

this standarf algebra may be generalized to $\mathrm{N}$ bodies

but

$$
\begin{aligned}
\sum_{1>f} \sigma_{i} \cdot \sigma_{j} & =\frac{1}{2}\left\{\sum_{1 j} \sigma_{1} \cdot \sigma_{j}-\sum_{i} \sigma_{1}^{2}\right\} \\
& =\frac{1}{2}\left\{\sum_{1} \sigma_{1} \zeta_{j} \sigma_{j}-\sum \sigma_{1}^{2}\right\}
\end{aligned}
$$




$$
\sum \sigma_{1}=2 S \quad S: \operatorname{total} \operatorname{spin}
$$

so

$$
\sum \sigma_{1} \cdot \sigma_{j}=\frac{1}{2}\left\{(2 S)^{2}-\sum \sigma_{i}^{2}\right\}
$$

and recall

$$
\sum \sigma_{1}^{2}=3 \mathrm{~N} \quad \mathrm{~N} \text { is the number of particles }
$$

so finally, the $\mathrm{N}$ body generalization is

$$
\begin{aligned}
{\left[\sigma_{i} \cdot \sigma_{j}\right.} & =\frac{1}{2}\left\{(2 S)^{2}-3 N\right\} \\
& =\frac{1}{2}\{4 S(S+1)-3 N\}
\end{aligned}
$$

This is an example of the group SU(2). With the example of angular momentum as a guide, we can now look at different groups. Let us begin by looking at the group of transformations
$u+d$
(or $\left.p_{+}^{+} n\right)$

there are four possible transformations

$\begin{array}{lll}u+d & \text { Number of transformations } \\ u+u & \text { identity: } & 1 \\ d+u & \text { operators: } & 3 \\ d+d & \text { total: } & 4\end{array}$


The number of operators for $S U(n)$ is given by $n^{2}-1$. This treatment may be generalized to $\mathrm{n}$ states. For example: quarks with spin

$$
u \uparrow \quad u \downarrow \quad d \uparrow d \psi
$$

if there are $n$ states:

$$
\begin{array}{ll}
n^{2} & \text { transitions } \\
1 & \text { is an identity } \\
n^{2}-1 & \text { operators }
\end{array}
$$

For all of these cases we can make the same kind of construction. By taking the sum of the squares of all of the generators, you get an operator like $\mathrm{J}^{2}$ that commutes with al1 the operators, and has elgenvalues determined by the appropriate Lie algebra. One can do this with SU(3). Begin with the generators of $\mathrm{SU}(3)$ and construct a quantity analogous to $\mathrm{J}^{2}$, and find Its eigenvalues. Structurally all of the groups work the same way in this respect. For SU(3)

$$
\begin{aligned}
U & =\frac{1}{2} \sum_{i j} \lambda_{i} \lambda_{j}\langle U\rangle-\frac{n}{z}\left\langle\lambda_{i} \cdot \lambda_{j}\right\rangle \sigma\left(r_{i j}\right\rangle \\
& =\frac{1}{2}\{C(N)-n C(3)\} U-\frac{n}{2} C(3)
\end{aligned}
$$

here $C(N)$ is the eigenvalue of the Casimir operator. The singlet state value For $\mathrm{C}$ is 0 . (Note: compare the expression above to the angular momentum case.) 


\subsection{Multiquark systems from hadrons}

How do we use our present knowledge? There are several multiquark systems to consider:

(1) " $\alpha$ " particle $4 \mathrm{q}$ all in the same s state 2-body interaction taken Erom ordinary hadrons.

(2) deuteron A weakly bound set of quarks, short-range potential.. a large fraction of the time, q's exist as mesons outside the range of the potential.

(3) $Q \equiv Q \quad q \quad$ A system of heavy quarks (Q) and Iight quarks (q). The dynamics may be separatıd, avolding problems with color.

Now let us consider these systems in greater detail.

" $\alpha$ " particle. Consideration of the $\alpha$ particle uses the dominance of the color magnetic field. The color electric fleld saturates and does not give strongly bound states. The color magnetic field is not saturated, and is large. For example:

$$
\begin{array}{ll}
\text { Color magnetic: } & \mathrm{M}_{\Delta}-\mathrm{M}_{\mathrm{N}}=300 \mathrm{MeV} \\
\text { Color electric: } & \text { Binding energy of }{ }^{2} \mathrm{H}=2.2 \mathrm{MeV}
\end{array}
$$

The color magnetic force results from one gluon exchange, while color electric forces require more gluons. In light of these considerations, it is reasonable to neglect the color electric force with respect to the color magnetic for $d$ 's and deuterons. Considet $N-N$ interactions. We observe:
a) Short-range repulsion
b) Long-range interaction described by one pion exchange (OPE)
c) Intermediate attraction 
OPE. One pion exchange is not described by six quark dynamics. A pion is a pion, whether we discuss quarks or not

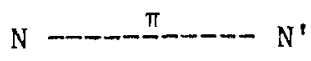

Repulsive core. Can the Pauli principle explain the repulst.ve core? If $\mathrm{p}$ and $\mathrm{n}$ are considered to be fundamental, the Pauli principle does not apply to them, as they are distinguishable particles. Perhaps though, by applying Pauli's principle to quarks that compose protons and neutrons, we can arrive at a repulsive core.

This question is an open one. Can the quan!itative behavior be explained? This question is important when considering six quark dibaryons. Intermediate attraction. Can quarks and a 2-body potential produce this interaction?.

The Feynman diagrams that we might consider are:

Direct Interaction:

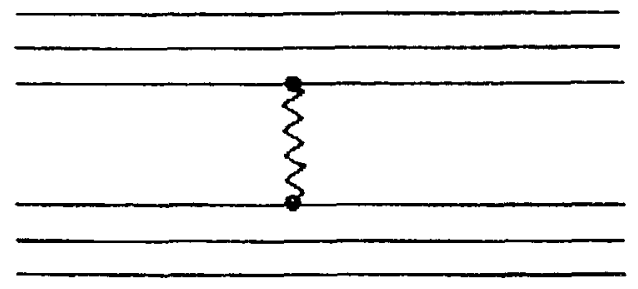

This diagram gives no contribution, (between color singlets - the gluon octet cannot couple)

\section{Two Gluons}

Two gluon exchange 


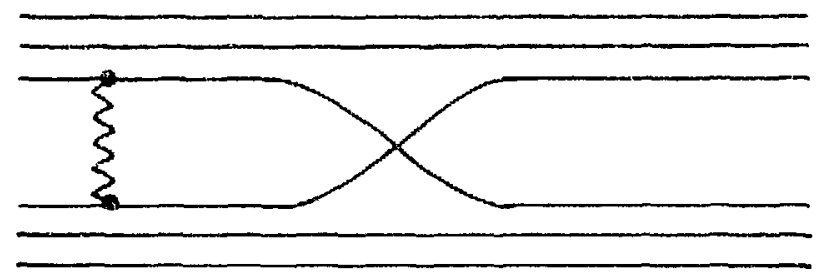
A one gluon exchange with
a quark exchange

The exchange interaction involves the overlap of wave functions, a nd does not glve long-range forces. It is sensitive to the wave function tails and is difficult to caiculate.

Now consider quark matter a particles (real $\alpha$ particles). $\alpha^{\prime} s$ are thought of as 4 nucleons, but could be considered a system of 12 quarks. The Pauli principle does not prevent this. But we must deal with Pauli, Heisenberg, and Fermt all at the same time (uncertainty principle, hyperfine splitting). Consider

\begin{tabular}{|c|c|c|c|c|}
\hline & Paul & & & Fermi \\
\hline $\begin{array}{r}u t \\
t\end{array}$ & $\begin{array}{r}\mathrm{u} \uparrow \\
+\end{array}$ & $\begin{array}{r}11 \uparrow \\
+\end{array}$ & color singlet & $\begin{array}{l}\Delta^{++}+ \\
\Delta^{++}+\end{array}$ \\
\hline $\begin{array}{r}\mathrm{d} \uparrow \\
+\end{array}$ & $d \uparrow$ & $d \uparrow$ & & $\Delta^{-} \uparrow$ \\
\hline
\end{tabular}

When we put everything together we get $\Delta^{\prime} \mathrm{s}$. It also costs us $1200 \mathrm{MeV}$ to put 4 nucleons together (by hyperfine). While $|n \uparrow\rangle$ and $|p \downarrow\rangle$ can occupy the same state, when we look at the quarks

$$
|d \uparrow d+u+\rangle|u+u+d \uparrow\rangle
$$


3 colors couple to a color singlet

$3 \mathrm{~d}$ 's antisymmetric in color

$\Delta^{-}$

3 U's antisymmetric in color

$\Delta^{++}$

We get $\Delta$ 's again. Only $1 / 9$ of the nucleon configurations in these states are permissible $-8 / 9$ are Paulf blocked. At short ranges, the two nucleon system is in the state

$|\Delta \Delta\rangle$

while at large I

$$
|\mathrm{pn}\rangle
$$

the total state is then given by

$$
\begin{aligned}
& x=f_{1}(r)|p n\rangle+f_{2}(r)|\Delta \Delta\rangle \\
& f_{1}(r)<<1 \quad \text { r close } \\
& f_{2}(r) \ll 1 \quad r \text { far }
\end{aligned}
$$

If we make $f_{2}(r)$ narrow, it costs us kinetic energy from

$$
\begin{aligned}
& \Delta x \Delta p \stackrel{=}{=} \\
& K E=\langle x|T| x\rangle=f_{2}^{*}(r) \frac{\partial^{2} f_{2}}{\partial r^{2}}
\end{aligned}
$$

this is a repulsive core type of Interaction. 
Several groups have tried to use simple potentials to calculate energies of. 6 quark systems (Maltman-Isgur). MI's calculation gives
a) 23-quark clusters (deuteron)
b) core
c) problematic Van der Waals

For a more preclse treatment, we will use the SU(2) p exchange model. To calculate the isospin magnetic interaction due to $\rho$ exchange, as before

$$
\begin{aligned}
U & =-\sum_{i>j}\left(\vec{\sigma}_{i} \cdot \vec{\sigma}_{j}\right)\left(\vec{\tau}_{i} \cdot \vec{\tau}_{j}\right) \\
& =-\sum_{i>j}\left(\sigma_{i}^{\alpha} \tau_{i}^{\beta}\right)\left(\sigma_{j}^{\alpha} \tau_{j}\right)
\end{aligned}
$$

(written to group the $i$ and $j$ operators). In the nucleon case, there are 4 possible states

$$
\mathrm{n}+\mathrm{n}+\mathrm{p} \uparrow \mathrm{p} \downarrow
$$

this leads to SU(4) with 15 generators. These generators are

$$
\text { transition }
$$
$3 \tau$
$n+p$

30

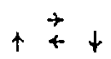

$9 \sigma \tau$

$\mathrm{n} \uparrow \rightarrow \mathrm{p} \downarrow$

spinflips

... all generators are composed of $\sigma$ and $\tau$ pieces. Using the $J^{2}$ analogy 


$$
\begin{aligned}
U & =-U\left[\sum_{i>j} g_{i}^{\alpha} g_{i}^{\alpha}-\sum_{i>j}\left(\stackrel{\vec{\sigma}}{j}_{i} \cdot \stackrel{+}{\sigma}_{j}\right)\left[\left(\vec{t}_{i} \cdot \vec{\tau}_{j}\right)\right]\right. \\
& =\frac{U}{2}\left[C_{4}(t o t)-N C_{4}(q)-C_{S}-C_{I}+N(\quad)\right] \\
S(S+1) & i(i+1)
\end{aligned}
$$

Now we go to color spin SU(6)

$$
\begin{aligned}
& \text { R t B } \downarrow \text { G } \uparrow \quad \text { (red, blue, green) } \\
& U=-\frac{U}{2}\left[C_{6}(t o t)-C_{3}(t o t)-\frac{8}{3} J(J+1)-16 N\right]
\end{aligned}
$$

Now to calculate the hyperfine interaction, plug in the C's. If we are looking at hadrons, the Casimir of SU(3) drops out, since hadrons are color singlets. For the $\Delta$, we have

symmetric in spin and isospin

antisymmetric in color spin

Using the Young tableaux for $\mathrm{SU}(6)$, we find

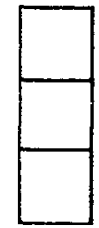

20 dimensional representation $\quad C(20)=42$

for the nucleons

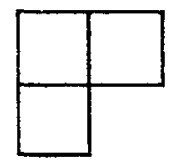

70 dimensional representation $C(70)=66$

The $\Delta-\mathrm{N}$ mass splitting is given by

$$
M_{\Lambda}-M_{N}=\frac{U}{2}(32)
$$


using the same technique for the $\alpha$, we get (note: a nice explanation of Young's tableaux is included in F. Close's book An Introduction to Quarks and Partons.)

$$
\begin{aligned}
& C_{6}: \text { singlet state } \\
& C_{3}: \text { singlet state } \\
& S: \quad 0 \\
& M(\alpha)=\frac{U}{2}(16 N) \\
& =M(4 \Delta)
\end{aligned}
$$

The most likely bound dibaryon is the dilambda (H particle). This is due to the maxlmization of $\mathrm{C}_{6}$ through the maximization of symmetry.

\begin{tabular}{|l|l|l|}
\hline$u$ & $d$ & 2 \\
\hline$u$ & 9 & 2 \\
\hline
\end{tabular}

- repeated flavors lower the Casimir

$$
M_{H}=-2 M_{\Lambda}=-\frac{1}{2}\left[M_{\Delta}-M_{N}\right]
$$

this overestimates the binding - it neglects su(3) breaking

$$
\begin{aligned}
& s-u \\
& u-u
\end{aligned}
$$


with a type of wave functions, $H$ is not bound, $T$ is too large. But this treatment has neglected a change in size...

change in size - $-\cdots+\quad$ lower energy $-\cdots+$ binding

The color magnetic force gives a weak binding. However, In such a weakly bound state, the constituents spend $70 \%$ of their time outside the range of the potential. Calculations using rigid confining bags are using the wrong wave function when they say the dibaryon is not bound. It costs some energy to keep the system in the bag all the time. Now let us summarize:

H-dibaryons: 6 quark systems. Bag and lattice calculations seem to conflict. The main difficulty is that all of the present descriptions are inadequate for weakly bound systems. The corstituents spend significant amounts of time outside the range of $V$. Some constraints are:

$$
\left(\frac{p^{2}}{2 \mu}+V\right) \psi=E \psi
$$

at larger $r$, where $V=0$

$$
\begin{aligned}
& \psi=\frac{e^{-K r}}{r} \quad K=\frac{m \cdot B E}{h^{2}}=200 \mathrm{MeV} \\
& B E \text { is the binding energy }
\end{aligned}
$$

Also, the wave functions, because of weak binding, must look like $2 \Lambda$ wave functions. 
8.2 Weakly bound two meson systems

Consider the Schroedinger equation for a weakly bound two-meson

system

$$
\begin{aligned}
& \left(\frac{p^{2}}{2 \mu}+V / m_{1} m_{2}\right) \psi=E \psi \quad \begin{array}{l}
m_{i}: \text { quark mass } \\
m_{u}=m_{d}=330 \mathrm{MeV}
\end{array} \\
& m_{s}=510 \mathrm{MeV} \\
& M_{k}=500 \mathrm{MeV} \\
& M_{\pi}=140 \mathrm{MeV}
\end{aligned}
$$

where we assume that the interaction potential is a short range effective hyperfine interaction inversely proportional to the product of quark masses

$$
\begin{aligned}
& 2 \mu=m=\text { meson mass } \\
& p^{2}+\frac{M_{m} V}{m_{l} m_{2}}=E M_{m} \psi
\end{aligned}
$$

Such an equation has a bound state if the function $\left(\frac{M_{1} V}{m_{1} m_{2}}\right)$ is sufficiently large. Since $V$ is unknown, we cannot give a quantitative estimate, we can compare different systems

$$
\begin{aligned}
& \text { For a } \pi \pi \text { system, } \mathrm{m}_{1}=\mathrm{m}_{2}=330, M=140 \\
& \frac{\mathrm{M}_{\mathrm{m}}}{\mathrm{m}_{1} \mathrm{~m}_{2}}=\frac{140}{(330)^{2}} \mathrm{~V}=1.29 \times 10^{-3} \mathrm{~V}
\end{aligned}
$$

for a $\overline{k K}$ system, $\mathbb{m}_{1}=510, \mathbb{m}_{2}=330, M=500$

$$
\frac{M_{m} V}{m_{1} m_{2}}=\frac{500}{(510)(330)}=2.97 \times 10^{-3} V
$$

This is the optimum value. For higher masses the value drops again. Two 


$$
\begin{aligned}
& \psi_{\mathrm{m}}(q \bar{q})=x_{\text {color }} \cdot \phi(x, \sigma \ldots) \\
& \psi_{B}(q q q)=x_{\text {color }} \cdot \phi(x \ldots)
\end{aligned}
$$

We cannot do this for more than 3 quarks though. We cannot write an effective potential. For example, take a $4 q$ system. The wave function of a $4 q$ systen is a $2 \times 2$ matrix in color space. This wave function is not gauge Invariant if we transform only the quarks. The requirement of transforming quarks and gluons makes writing an effective interaction difficult. Even a confining potential is unphysical since as q's are separated, a $q \bar{q}$ pair is formed.

\section{A Study of Multiquark Systems}

So there are many difficulties involved in extrapolating what we know in the $q \bar{q}$ and $q q q$ cases to higher number mult1quark systems. There are new effects that we cannot calculate. Now we w111 try to overcome some of these difficulties to get quantitative or semiquantitative results. First, let us begin by reviewing why mitiquark systems are exotic and weakly bound. To do this let us begin with a potential model that is like the $\rho$ exchange model formerly used for the deuteron. The potential is of the form

$$
\begin{aligned}
U= & \sum \lambda_{1} \lambda_{j} U\left(r_{i j}\right) \\
\langle U\rangle & =\sum_{1\rangle_{j}} \lambda_{1} \cdot \lambda_{j}\left\langle U\left(r_{1 j}\right)\right\rangle \\
& =\frac{1}{2} C(N)-n C(3)
\end{aligned}
$$

where $\lambda$ 's are the operators for color (analogous to $\sigma^{\prime} s$ for angular momentum), $C$ is a constant, $n$ is the number of particles. 
What we obtain from such a potential model is a bind: ng energy that is proportional to the number of particles, so the mass of a hadron is proportional to the number of quarks. This result was first found by Nambu. Nambu supposed that hadrons are bound by one gluon exchange, and arrived at: the hadron mass formula:

$$
\begin{aligned}
& M(H)=a_{0} \eta_{q}(H) \\
& M(q \bar{q})=2 m_{0}
\end{aligned}
$$$$
\text { Any multiquark system will simply break up into }
$$$$
\text { a system of } 3 q \text { and } q \bar{q} \text { sets. }
$$

Before going onto look at more multiquark systems, it is useful to review some angular momentum algebra. Recal1:

$$
\begin{aligned}
& \mathrm{J}^{2}=\mathrm{J}_{\mathrm{x}}^{2}+\mathrm{J}_{\mathrm{y}}^{2}+\mathrm{J}_{\mathrm{z}}^{2} \\
& \mathrm{~J}_{\psi}^{2}=\mathrm{j}(\mathrm{j}+1) \psi \quad \mathrm{J} \text { is the total angular momentum } \mathrm{J}_{\mathrm{T}}=\mathrm{J}_{1}+\mathrm{J}_{2} \\
& \mathrm{~J}_{1} \cdot \mathrm{J}_{2}=\frac{1}{2}\left[\mathrm{~J}_{\mathrm{T}}^{2}-\mathrm{J}_{1}^{2}-\mathrm{J}_{2}^{2}\right] \\
& =\frac{1}{2}\left[\mathrm{~J}_{\mathrm{T}}\left(\mathrm{j}_{\mathrm{T}}+1\right)-\mathrm{j}_{1}\left(\mathrm{j}_{1}+1\right)-\mathrm{j}_{2}\left(\mathrm{j}_{2}+1\right)\right]
\end{aligned}
$$

this standard algebra may be generalized to $N$ bodies

but

$$
\begin{aligned}
\sum_{1>j} \sigma_{1} \cdot \sigma_{j} & =\frac{1}{2}\left\{\sum_{i j} \sigma_{i} \cdot \sigma_{j}-\sum_{i} \sigma_{i}^{2}\right\} \\
& =\frac{1}{2}\left\{\sum_{1} \sigma_{1} \sum_{j} \sigma_{j}-\sum \sigma_{1}^{2}\right\}
\end{aligned}
$$




$$
\sum \sigma_{i}=2 S \quad S: \text { total spin }
$$

so

$$
\sum \sigma_{i} \cdot \sigma_{j}=\frac{1}{2}\left\{(2 S)^{2}-\sum \sigma_{i}^{2}\right\}
$$

and recall

$$
\sum \sigma_{1}^{2}=3 \mathrm{~N} \quad \mathrm{~N} \text { is the number of particles }
$$

so finally, the $\mathrm{N}$ body generalization is

$$
\begin{aligned}
{\left[\sigma_{i} \cdot \sigma_{j}\right.} & =\frac{1}{2}\left\{(2 S)^{2}-3 N\right\} \\
& =\frac{1}{2}\{4 S(S+1)-3 N\}
\end{aligned}
$$

This is an example of the group SU(2). With the example of angular momentum as a guide, we can now look at different groups. Let us begin by looking at the group of transformations
$u_{+}^{+} \mathrm{d}$
$\left(\right.$ or $\left.p_{+}^{+} n\right)$

there are four possible transformations
$u+d$
Number of transformations
$u+u$
identity: $\quad 1$
$d+u$
operators: 3
$d+d$
total:
4 
The number of operators for $S U(n)$ is given by $n^{2}-1$. This treatment may be generalized to $n$ states. For example: quarks with spin

$$
u+u+d t d t
$$

if there are $n$ states:

$$
\begin{array}{ll}
n^{2} & \text { transitions } \\
1 & \text { is an identity } \\
n^{2}-1 & \text { operators }
\end{array}
$$

For all of these cases we can make the same kind of construction. By taking the sum of the squares of all of the generators, you get an operator like $\mathrm{J}^{2}$ that commutes with all the operators, and has elgenvalues determined by the appropilate Lie algebra. One can do this with SU(3). Begin with the generators of $\mathrm{SU}(3)$ and construct a quantity analogous to $\mathrm{J}^{2}$, and find its eigenvalues. Structurally all of the groups work the same way in this respect. For SU(3)

$$
\begin{aligned}
U & =\frac{1}{2} \sum_{1 j} \lambda_{i} \lambda_{j}\langle U\rangle-\frac{n}{z}\left\langle\lambda_{i} \cdot \lambda_{j}\right\rangle \sigma\left(r_{i j}\right\rangle \\
& =\frac{1}{2}\{C(N)-n C(3)\} U-\frac{n}{2} C(3)
\end{aligned}
$$

here $C(N)$ is the elgenvalue of the Casimir operator. The singlet state value for $\mathrm{G}$ is 0 . (Note: compare the expression above to the angular momentum case.) 


\subsection{Multiquark systems from hadrons}

How do we use our present knowledge? There are several multiquark systems to consider:

(1) " $\alpha$ " particle $4 \mathrm{q}$ all in the same s state 2-body interaction taken from ordinary hadrons.

(2) deuteron A weakly bound set of quarks, short-range potential.. a large fraction of the time, $q$ 's exist as mesons outside the range of the potential.

(3) $Q \equiv Q \quad q$ A system of heavy quarks (Q) and light quarks ( $Q$ ). The dynamics may be separated, avoiding problems with color.

Now let us consider these systems in greater detall. " $\alpha$ " particle. Consideration of the $\alpha$ particle uses the dominance of the color magnetic field. The color electric field saturates and does not give strongly bound states. The color magnetic field is not saturated, and is large. For example:

$$
\begin{array}{ll}
\text { Color magnetic: } & M_{\Delta}-M_{N}=300 \mathrm{MeV} \\
\text { Color electric: } & \text { Binding energy of }{ }^{2} \mathrm{H}=2.2 \mathrm{MeV}
\end{array}
$$

The color magnetic force results from one gluon exchange, while color electric forces require more gluons. In light of these considerations, it is reasonable to neglect the color electric force with respect to the color magnetic for $d^{\prime} s$ and deuterons. Consider $\mathrm{N}-\mathrm{N}$ interactions. We observe:
a) Short--range repulsion
b) Long-range interaction described by one pion exchange (OPE)
c) Intermediate attraction 
OPE. One pion exchange is not described by six quark dynamics. A pion is a pion, whether we discuss quarks or not

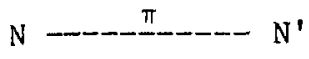

Repulstve core. Can the Pauli principle explain the repulstve core? If $p$ and $n$ are considered to be fundamental, the Paull principle does not apply to them, as they are distinguishable particles. Perhaps though, by applying Pauli's principle to quarks that compose protons and neutrons, we can arrive at a repulsive core.

This question is an open one. Can the quaritative behavior be explained? This question is important when considering six quark dibaryons. Intermediate attraction. Can quarks and a 2-body potential produce this interaction?.

The Feynman diagrams that we might consider are:

Direct Interoction:

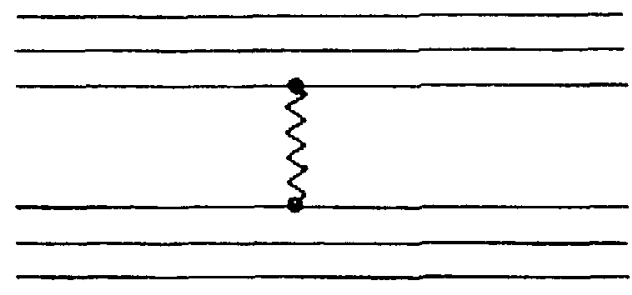

This diagram gives no contribution, (between color singlets - the gluon octet cannot couple)

Two Gluons

Two gluon exchange 
Exchange interaction

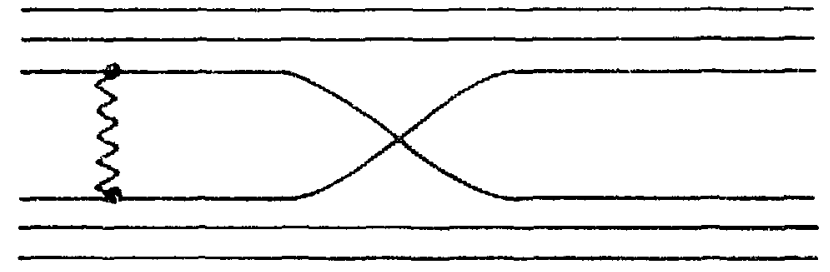

A one gluon exchange with

a quark exchange

The exchange interaction involves the overlap of wave functions, a nd does not give long-range forces. It is sensitive to the wave function tails and is difficult to calculate.

Now consider quark matter $\alpha$ particles (real $\alpha$ particles). a's are thought of as 4 nucleons, but could be considered a system of 12 quarks. The Pauli principle does not prevent this. But we must deal with Pauli, Heisenberg, and Fermi all at the same time (uncertainty principle, hyperfine splitting). Consider

Pauli

$u \uparrow$

$\uparrow$

$u \uparrow$
+

$\mathbf{u} \uparrow$

color singlet

$\begin{array}{rrr}d \uparrow & d \uparrow & d \uparrow \\ + & + & \downarrow\end{array}$
Fermi

$\Delta^{++}+$

$\Delta^{-} \uparrow$

$\Delta \downarrow$

When we put everything together we get $\Delta^{\prime} s$. It also costs us $1200 \mathrm{MeV}$ to put 4 nucleons together (by hyperfine). While $|n \uparrow\rangle$ and $|p t\rangle$ can occupy the same state, when we look at the quarks

$$
|d \uparrow d \uparrow u \downarrow\rangle
$$


3 colors couple to a color singlet

$3 \mathrm{~d}^{\prime} \mathrm{s}$ antisymmetric in color $\Delta^{-}$

3 U's antisymmetric in color $\Delta^{++}$

We get $\Delta^{\prime} s$ agaln. On $2 y 1 / 9$ of the nucleon configurations in these states are permisstble $-8 / 9$ are Paull blocked. At short ranges, the two nucleon system is in the state

$|\Delta \Delta\rangle$

while at large $r$

$|\mathrm{pn}\rangle$

the total state is then given by

$$
\begin{array}{ll}
x=E_{1}(r)|p n\rangle & +F_{2}(r)|\Delta \Delta\rangle \\
F_{1}(r) \ll 1 \quad r \text { close } \\
\mathrm{F}_{2}(r) \ll 1 \quad \text { r far }
\end{array}
$$

If we make $E_{2}(r)$ narrow, it costs us kinetic energy from

$$
\begin{aligned}
& \Delta x \Delta \mathrm{p} \cong \nVdash \\
& K E=\langle x|T| x\rangle=F_{2}^{*}(r) \frac{\partial^{2} f_{2}}{\partial r^{2}}
\end{aligned}
$$

this is a repulsive core type of interaction. 
Several groups have tried to use simple potentials to calculate energies of 6 quark systems (Maltman-Isgur). MI's calculation gives
a) 3-quark clusters (deuteron)
b) core
c) problematic Van der Waals

For a more precise treatment, we will use the SU(2) p exchange model. To calculate the isospin magnetic interaction due to $\rho$ exchange, as before

$$
\begin{aligned}
U & =-\sum_{i>j}\left(\vec{\sigma}_{i} \cdot \vec{\sigma}_{j}\right)\left(\vec{\tau}_{i} \cdot \vec{\tau}_{j}\right) \\
& =-\sum_{\substack{i>j \\
\alpha \beta}}\left(\sigma_{i}^{\alpha} \tau_{i}^{\beta}\right)\left(\sigma_{j}^{\alpha} \tau_{j}\right)
\end{aligned}
$$

(written to group the $i$ and $j$ operators). In the nucleon case, there are 4 possible states

$$
n \uparrow \quad n+p \uparrow p \downarrow
$$

this leads to SU(4) with 15 generators. These generators are

$$
\begin{aligned}
& \text { transition } \\
& 3 \tau \quad n+p \\
& 3 \sigma \\
& +\stackrel{+}{t}+ \\
& 9 \text { oT } \\
& \mathrm{n} \uparrow \rightarrow \mathrm{p} \downarrow \\
& \text { spinflips }
\end{aligned}
$$

... all generators are composed of $\sigma$ and $\tau$ pieces. Using the $\mathrm{J}^{2}$ analogy 


$$
\begin{aligned}
U & =-U\left[\sum_{i>j} g_{i}^{\alpha} g_{i}^{\alpha}-\sum_{i>j}\left(\dot{\sigma}_{i} \cdot \vec{\sigma}_{j}\right)\left[\left(\vec{\tau}_{i} \cdot \vec{\tau}_{j}\right)\right]\right. \\
& =\frac{U}{2}\left[C_{4}(t o t)-N_{4}(q)-C_{S}-C_{I}+N(\quad)\right] \\
s(s+1) & i(i+1)
\end{aligned}
$$

Now we go to color spin $\mathrm{SU}(6)$

$$
\begin{aligned}
& R \uparrow \downarrow B \uparrow \downarrow G \uparrow \downarrow \quad \text { (red, blue, green) } \\
& U=-\frac{U}{2}\left[C_{6}(\text { tot })-C_{3}(t o t)-\frac{8}{3} J(J+1)-16 N\right]
\end{aligned}
$$

Now to calculate the hyperfine Interaction, plug in the C's. If we are looking at hadrons, the Casinir of su(3) drops out, since hadrons are color singlets. For the $\Delta$, we have

symmetric in spin and isospin

antisymmetric in color spin

Using the Young tableaux for SU(6), we find

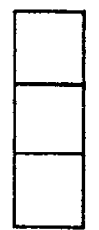

20 dimensional representation $\mathrm{C}(20)=42$

for the nucleons

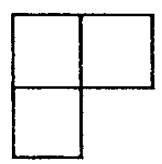

70 dimensional representation $C(70)=66$

The $\Delta-N$ mass splttting is given by

$$
M_{\Lambda}-M_{N}=\frac{U}{2}(32)
$$


using the same technique for the $\alpha_{2}$ we get (note: a nice explanation of Young's tableaux is included in F. Close's book An Introduction to Quarks and Partons.)

$$
\begin{aligned}
& \mathrm{C}_{6}: \text { singlet state } \\
& \mathrm{C}_{3}: \text { singlet state } \\
& \mathrm{S}: \quad 0 \\
& M(\alpha)=\frac{U}{2}(16 \mathrm{~N}) \\
& =M(4 \Delta)
\end{aligned}
$$

The most likely bound dibaryon is the dilambda ( $H$ particle). This is due to the maximization of $C_{6}$ through the maximization of symmetry.

\begin{tabular}{|l|l|l|}
\hline$u$ & $d$ & $s$ \\
\hline$u$ & $d$ & $s$ \\
\hline
\end{tabular}

- repeated flavors lower the Casimir

$$
M_{H}=-2 M_{\Lambda}=-\frac{1}{2}\left[M_{\Delta}-M_{N}\right]
$$

this overestimates the binding - it neglects SU(3) breaking

$$
\begin{aligned}
& s-u \\
& u-u
\end{aligned}
$$


with a type of wave functions, $H$ is not bound, $T$ is too large. But this treatment has neglected a change in size...

$$
\text { change in size - ---+ lower energy ---+ binding }
$$

The color magnetic force gives a weak binding. However, in such a weakly bound state, the constituents spend $70 \%$ of their time outside the range of the potential. Calculations using rigid confining bags are using the wrong wave function when they say the dibaryon is not bound. It costs some energy to keep the system in the bag all the t1me. Now let us summarize:

H-dibaryons: 6 quark systems. Bag and lattice calculations seem to conflict. The main difficulty is that all of the present descriptions are inadequate for weak1y bound systems. The consticuents spend significant amounts of time outside the range of $V$. Some constraints are:

$$
\left(\frac{p^{2}}{2 \mu}+V\right) \psi=E \psi
$$

at larger $r$, where $V=0$

$$
\psi=\frac{e^{-K r}}{r} \quad K=\frac{m \cdot B E}{k^{2}}=200 \mathrm{MeV}
$$

$B E$ is the binding energy

Also, the wave functlons, because of weak blnding, must look 11 ke $2 \Lambda$ wave functions. 


\subsection{Weakly bound two meson systems}

Consider the Schroedinger equation for a weakly bound two-meson system

$$
\begin{aligned}
& \mathrm{m}_{i} \text { : quark mass } \\
& \mathrm{m}_{\mathrm{u}}=\mathrm{m}_{\mathrm{d}}=330 \mathrm{MeV} \\
& \left(\frac{\mathrm{p}^{2}}{2 \mu}+\mathrm{V} /_{\mathrm{m}_{1} \mathrm{~m}_{2}}\right) \psi=\mathrm{E} \psi \\
& \mathrm{m}_{\mathrm{s}}=510 \mathrm{MeV} \\
& \mathrm{M}_{\mathrm{k}}=500 \mathrm{MeV} \\
& M_{\pi}=140 \mathrm{MeV}
\end{aligned}
$$

where we assume that the interaction potential is a short range effective hyperfine Interaction inversely proportional to the product of quark masses

$$
\begin{aligned}
& 2 \mu=m=\text { meson mass } \\
& p^{2}+\frac{M V}{m_{1} m_{2}}=E M_{m} \psi
\end{aligned}
$$

Such an equation has a bound state if the function $\left(\frac{M^{m}}{m_{1} m_{2}}\right)$ is sufficiently large. Since $V$ is unknown, we cannot give a quantitative estimate, we can compare different systems

$$
\begin{aligned}
& \text { For a } \pi \pi \text { system, } \mathrm{m}_{1}=\mathrm{m}_{2}=330, \mathrm{M}=140 \\
& \frac{\mathrm{M}_{\mathrm{m}}}{\mathrm{m}_{1} \mathrm{~m}_{2}}=\frac{140}{(330)^{2}} \mathrm{~V}=1.29 \times 10^{-3} \mathrm{~V}
\end{aligned}
$$

for a $\overline{K K}$ system, $\mathrm{m}_{1}=510, \mathrm{~m}_{2}=330, \mathrm{M}=500$

$$
\frac{M_{m} V}{m_{1} m_{2}}=\frac{500}{(510)(330)}=2.97 \times 10^{-3} v
$$

This is the optimum value. For higher masses the value drops again. Two 
to calculate $\left(\frac{g_{A}}{g_{V}}\right)_{N}$ we use many of the tricks involved in calculating the magnetic moments

$$
\begin{aligned}
\left(\frac{g_{A}}{g_{V}}\right)_{N} & =\frac{\left\langle p t\left|Z_{z} s_{+i}\right| n \uparrow\right\rangle}{\left\langle p\left|t_{t i}\right| n\right\rangle} \\
& =\frac{\left\langle p \uparrow\left|S_{z} t_{+}\right| p \uparrow\right\rangle}{1 / 2} \\
& =\frac{\left\langle p \uparrow\left|\left(\vec{S}_{u}+\vec{S}_{d}\right)\left(\vec{S}_{u}-\vec{S}_{d}\right)\right| p \uparrow\right\rangle}{3 ! 4} \\
& =\frac{2-3 / 4}{3 / 4}=5 / 3
\end{aligned}
$$

This is the wrong value to be quoted as coming from the quark model. We cheated $\ldots$ we assumed that

$$
\left(\frac{\mathrm{B}_{\mathrm{A}}}{\mathrm{B}_{\mathrm{V}}}\right)_{\mathrm{q}}=1
$$

for a nucleon, the expression is

$$
\left(\frac{g_{A}}{g_{V}}\right)=\left(\frac{g_{A}}{g_{V}}\right) \cdot \frac{5}{3}
$$

in this case we have one piece of data, and one free parameter. Electroweak theory says that $\left(\frac{g_{A}}{g_{V}}\right)_{q}=1$, but this is not for the same quarks. At present, we can discuss two models: 
Quark-Parton

We know the constituent parts (quark)

but not the hadron wave functions.

Structure functions are measured to

get a measure of the nucleon wave

functions.

Quarks behave as pointlike particles

in deep inelastic scattering. There is GWS coupling.
Quasinuclear with Color

We know the hadronic wave functions but not the constituent parts. Po tential models give wave functions exactly. We do not know quark masses interactions...

Hadronic wave functions are given by simple potential model calculations.

QCD should provide the connection between these models. There has not been much communication between them yet. The question to ask now is "What more can we learn?" We might look at $g_{A}$. To do this, begin by noting the property

$$
\left[\sum_{z i} S_{+i}, \quad \sum 2 S_{z j} t_{-j}\right]=\sum_{i} t_{z i}-2 T z=1
$$

now look at the element

$$
\sum_{x}\left\langle p\left|\sum_{1} z S_{z i}{ }^{t}+i\right| x\right\rangle\left\langle x\left|\sum_{k} z S_{z j} t_{-j}\right| p\right\rangle
$$

where the $\mathrm{x}$ sums over nuclear states

$$
\left.\mathrm{g}_{\mathrm{A}}^{2}=1-\sum_{\mathrm{x} \neq \mathrm{h}}\left\langle\mathrm{p}\left|\mathrm{t}_{+}\right| \mathrm{x}\right\rangle\left\langle\mathrm{x}\left|\mathrm{t}_{-}\right| \mathrm{p}\right\rangle+\sum_{\mathrm{x}}\left\langle\mathrm{p}\left|\mathrm{t}_{-}\right| \mathrm{x}\right\rangle *_{\mathrm{x}}\left|\mathrm{t}_{+}\right| \mathrm{p}\right\rangle
$$

in this sum, only the $\Delta$ contributes

$$
g_{A}^{2}=5^{2} / 3
$$


Adler and others used experimental values to calculate the sum of the elements above. We need to be able to calculate matrix elements between $p$ and $n$, and we should also be able to get the excited state contributions. More advanced treatments of this problem are examples of the use of baryon spectroscopy. Modelers can always bring the value of $\left(\mathrm{g}_{\mathrm{A}}^{2}\right)$ down. This in itself is not particularly slgnificant. However, if model can also be used to predict excited states, the it will have achieved something significant. A typical problem that you would like to describe is that of pion-nucleon scattering near a resonance. There are two models that we might compare and constrast. They are the quark-proton model and the quasinuclear model. The quark-proton model is more fundamental, but it gives us no way of getting hadronic properties. On the other hand, the quasinuclear model gives the hadronic properties, but does not address the question of what are the fundamental constituents. There are several considerations:

1) Dressed quarks? Should we use an approach, analogous to constituent electrons in an atom with a Coulomb fleld, where we look at each valence quark, including its portion of the sea quarks and glue?

2) Lortutz invariance. The question to be answered is how large is the ocean of $4 \bar{q}$ pairs? It is not Lorentz invariant, and a simple diagram illustrates this.

The production of sea quarks might be diogrammed os

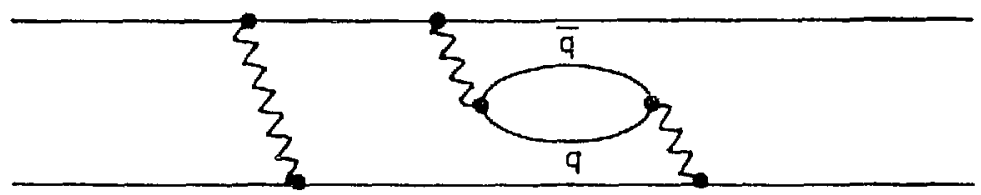
or alternotely as

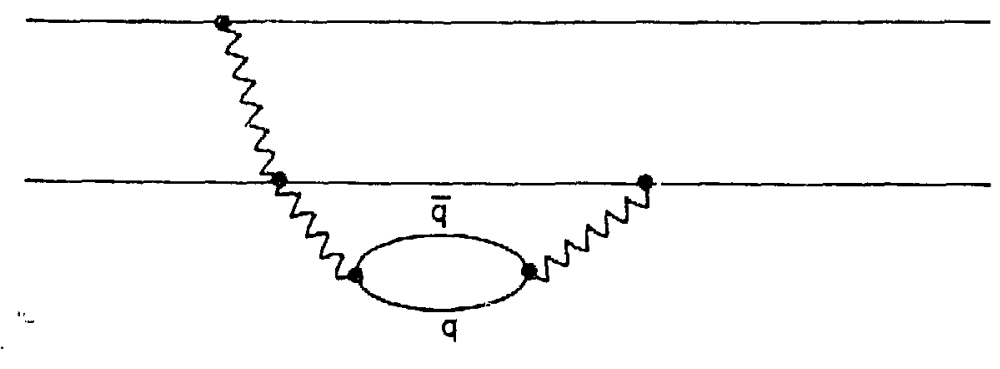


by looking at the diagram below we can see the problems of Lorentz Invarlance

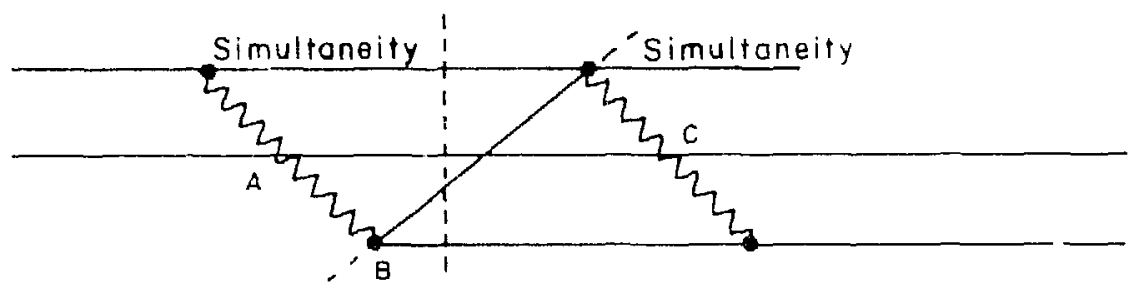

A gluon emitted at $A$ produces a $q \bar{q}$ palr at $B$. One of the produced quarks annihilites with one of the origlnal quarks at $C$, producing a gluon. By taking different lines of simultaneity we get different pictures of how many quarks there are. If the lines of simultanelty are vertical, there is a span of time where there are more than two quarks. If we take a diagonal line of simultaneity, there is never more than one quark. This demonstrates the problem of Lorentz Invariance for sea quarks.

\section{Exotics - A Continuation}

It is lnieresting to consider the meson spectrum and possible states calied glueballs. When we have Alscussed hadrons, we have talked only of consitituent quarks. But hadrons really consist of qq̄ pairs and gluons as well as $3 q$ systems. Some have talked in a similar way about constituent gluons being bound together by exchanging gluons. The idea of bound glue comes directly from the properties of SU(3) non-abelian gauge theorels. A Feynman diagram describing bound glue of ght be

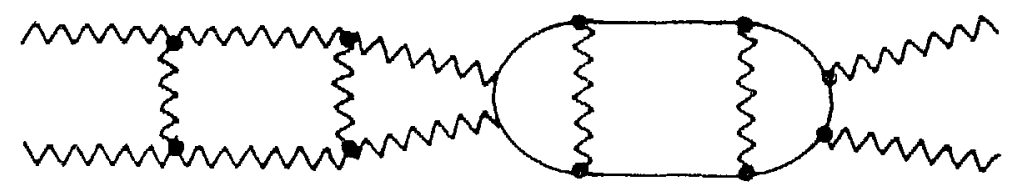

Recall that gluons may produce q $\bar{q}$ palrs. But how might such states be seen? 
One way of trying to observe exotic states is to try to find certain particles that may only be constructed via a certain process. For example:

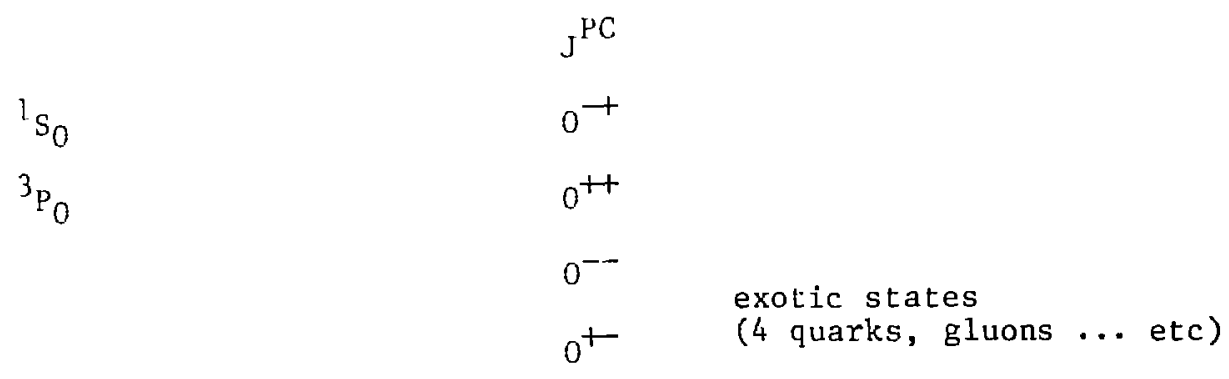

We should look to the mass spectra for exotic states. To do this, we might go back to quarkonium and look at its spectrum first, for we need to understand the mass spectrum before we can pick out the exotics.

The best data for quarkonium energy levels comes from the heavy quarks, where the spectrum really looks like hydrogen energy levels.

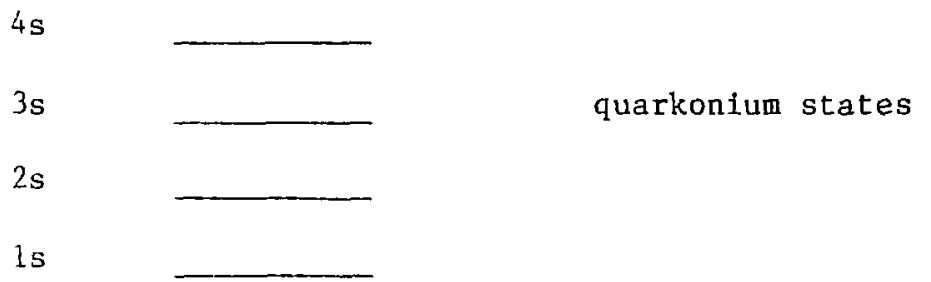

The question we now address is why the $c \bar{c}$ (charmonium) states are narrow while the $p$ is broad?

Note: Since the uncertainty principle states $\Delta E \Delta t=h / 2$, the width of a state in energy is an indication of its lifetime $\mathrm{T}_{\mathrm{L}} \cong \frac{h}{\mathrm{Z \Delta E}}$, and therefore the rate at which a particular process proceeds. Broad states (large $\Delta \mathrm{E}$ ) indicate a short lifetime, while narrow states (sma11 $\Delta E$ ) indicate a longer lifetime.

To begin considering the $c \bar{c}$ and $p$, we recall the OZI rule (Okubo 1963, Zweig 
1964, Iizuka 1966). The OZI rule states that processes represented by disconnected diagrams are greatly suppressed. The $\rho$ decay is given by

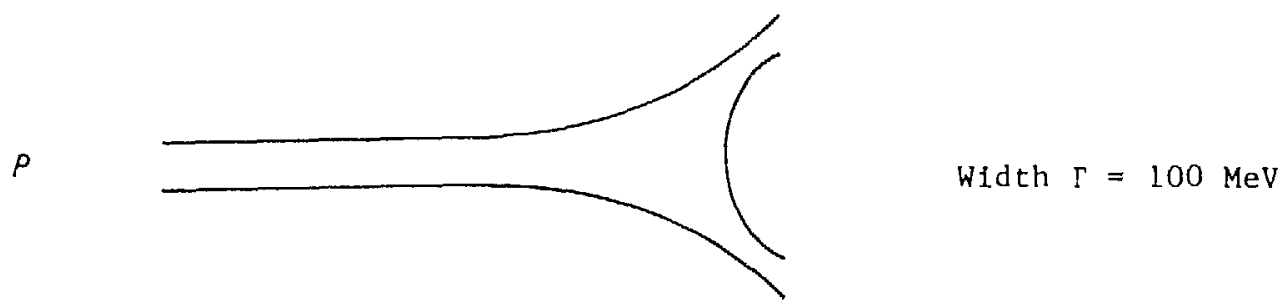

The $\vec{c} \vec{c}$ decay that is favored, but forhidden by energy conservation is

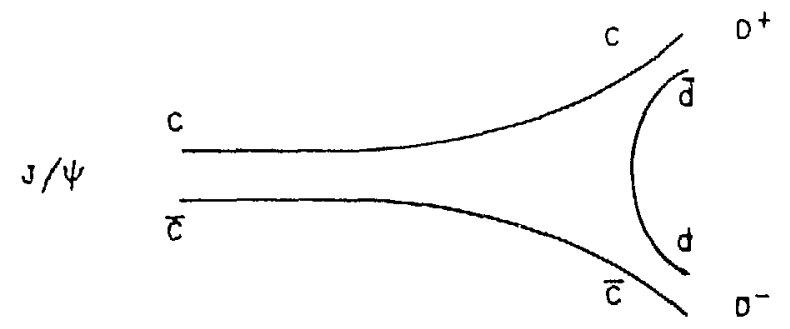

This diagram would be favored if it were not forbidden by energy conservation.

This leaves us with the next diagram

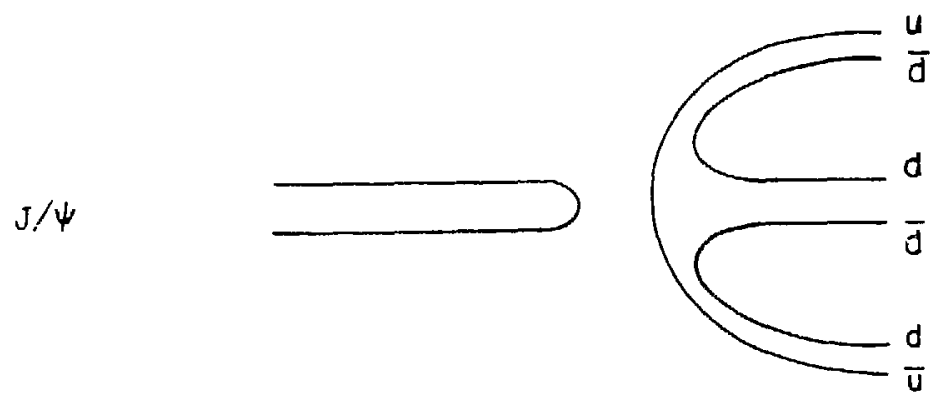

This process for $c \vec{c}$ decay is strongly suppressed because of the OZI rule - it is a disconnected diagram. The strong suppression leads to a narrow width of $\Gamma=0.063 \mathrm{MeV}$. The OZI rule also explains $\phi$ decay. Two possible schemes are
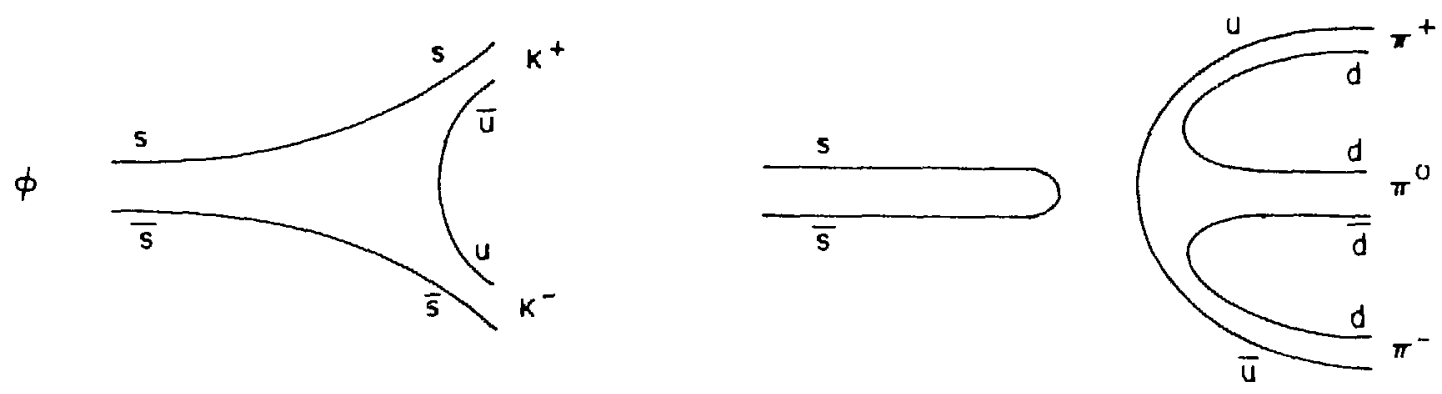
Phase space considerations favor the process on the right, but the ozI rule suppresses this process. The process on the left, $\phi+\mathrm{K}^{+} \mathrm{K}^{-}$proceeds as the dominant one. (Ref. Perkins, Introduction of High Energy Physics).

We might ask why the OZI rule is true. Vector meson decays described by unconnected diagrams require the production of at least 3 gluons to conserve color and charge configuration. This suppresses the process by $8^{3}$ where $g$ is the strong coupling constant. The gluons carry 4 momentum and as the $q$ masses get larger, the coupling constants g get smaller (asymtotic freedom) so the states get natrower. Suppression grows as $\mathrm{g}^{3}$.

All of this discussion is fine unti? you try to make it quantitative. Take the following example that illustrates the problem. Why is $\phi+\eta \pi$ forbidden since $d+\mathrm{K}^{+} \mathrm{K}^{-}$, why can't the process be

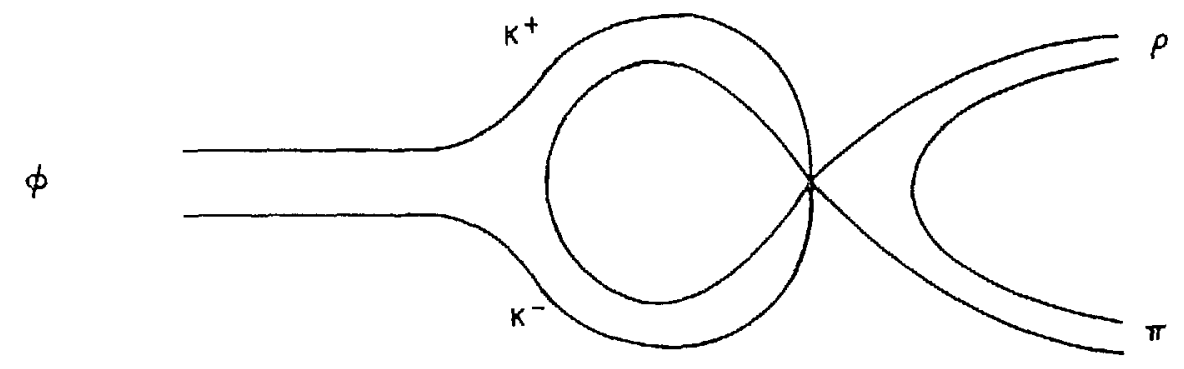

How is it that the $\mathrm{K}^{+} \mathrm{K}^{-}$pair remembers that it came from a $\phi$ ? Flavor disappearance by annihilation requires 2 or 3 gluons.

\subsection{Flavor mixing}

Now consider how flavors of quarks might mix. Nine states may be make Erom

$\begin{array}{cc}u & \bar{u} \\ d & \text { d } \\ s & \bar{s}\end{array}$

Since $u \bar{i}, \vec{s} \vec{s}$ and $d \bar{d}$ all have the same quantum numbers, they can mix. 


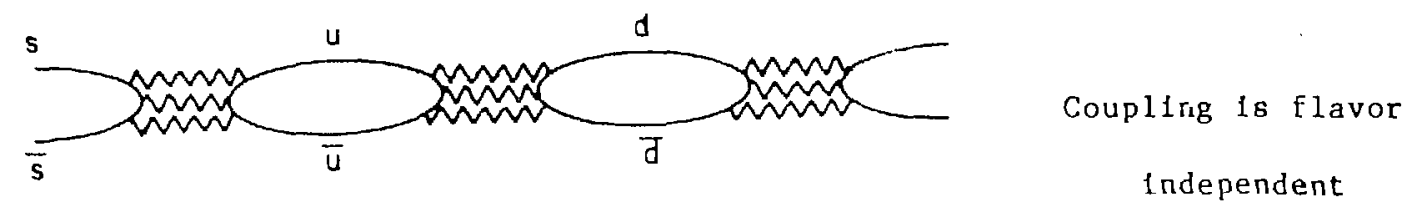

(similar to a nuclear bubble diagram)

This state may be described

$$
|\psi\rangle=\left|\psi_{0}\right\rangle+\sum_{i}|1\rangle \frac{\langle i|v| 0\rangle}{E_{0}-E_{i}}
$$

A family of particles results from the specific mixing of flavors. We can tabulate how the mixture produces each family

\begin{tabular}{lccc}
\hline Mixture & Pseudoscalar & Vector & Tensor \\
\hline$\frac{1}{\sqrt{2}}\left(\mathrm{uu}_{\mathrm{u}} \mathrm{d} \overline{\mathrm{d}}\right)$ & $\pi^{0}$ & $0^{0}$ & $\mathrm{A2}{ }^{0}$ \\
$\frac{1}{\sqrt{2}(\mathrm{uu}+\mathrm{d} \overline{\mathrm{d}})}$ & $n$ & $\omega$ & $\mathrm{f}^{0}$ \\
$\mathrm{ss}^{-}$ & $n^{\prime}$ & & \\
\hline
\end{tabular}

Adding the $s$ quark costs some energy because oit the $s$ mass. $\phi+\rho \pi$ is Forbidden because $\phi$ is a vector particle, $f^{\prime}+\pi \pi$ is Forbidden because $f^{\prime}$ is a tensor particle. Things are different for the pseudoscalars. They vary by a 2 gluon exchange, and the 2 gluon state 1 s stronger.

After our long discussion of quarkonium spectra, decay processes, flavor mixing, we return to quarkonium. We can look at mixing in the spectra 


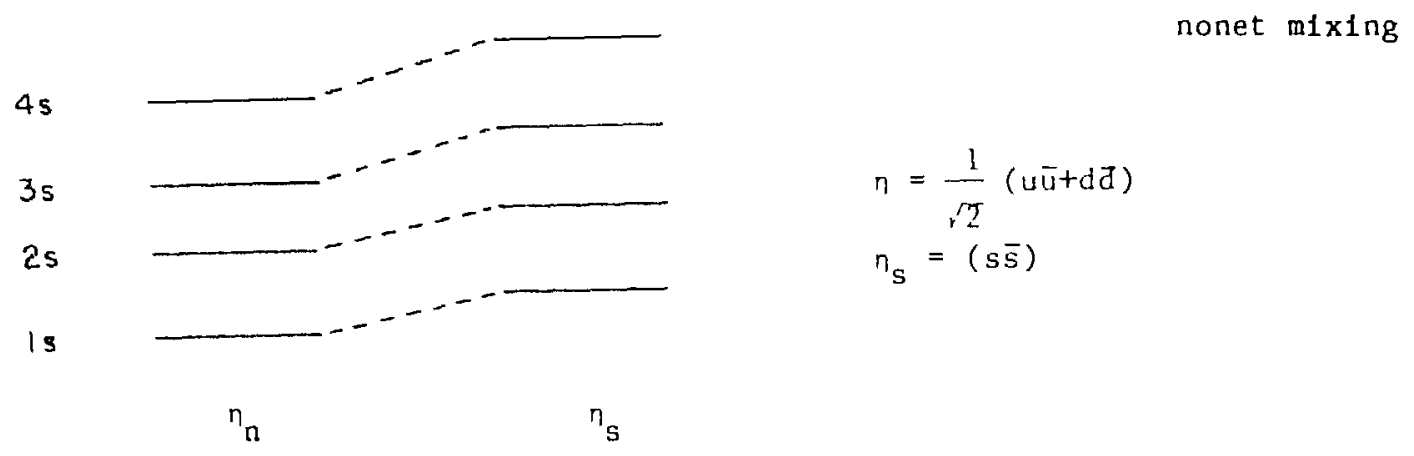

The elgenvalues actually lie in-between the two states. To really do this problem correctly, we must know
1) Nonperturbative solutions of $n_{n}$ and $n_{s}$
2) The interactions
3) Radial excitations and wave functions

Care must be taken to dolng this calculation since it includes a model dependent cutoff.

I appreclate very much the help of Christopher Fasano in preparing the lecture notes. 